\title{
Search for cold and hot gas in the ram pressure stripped Virgo dwarf galaxy IC $3418^{\star, \star \star}$
}

\author{
P. Jáchym ${ }^{1,2}$, J. D. P. Kenney ${ }^{2}$, A. Ružička ${ }^{1}$, M. Sun ${ }^{3}$, F. Combes ${ }^{4}$, and J. Palouš ${ }^{1}$ \\ 1 Astronomical Institute, Academy of Sciences of the Czech Republic, Boční II 1401, 14100 Prague, Czech Republic \\ e-mail: jachym@ig.cas.cz \\ 2 Department of Astronomy, Yale University, 260 Whitney Ave., New Haven, CT 06511, USA \\ e-mail: jeff.kenney@yale.edu \\ 3 Eureka Scientific, 2452 Delmer Street Suite 100, Oakland, CA 94602, USA \\ e-mail: mingsun.cluster@gmail.com \\ 4 Observatoire de Paris, LERMA, 61 Av. de l'Observatoire, 75014 Paris, France \\ e-mail: francoise.combes@obspm.fr
}

Received 4 October 2012 / Accepted 30 April 2013

\begin{abstract}
We present IRAM 30 m sensitive upper limits on CO emission in the ram pressure stripped dwarf Virgo galaxy IC 3418 and in a few positions covering $\mathrm{H}$ II regions in its prominent $17 \mathrm{kpc} \mathrm{UV} / \mathrm{H} \alpha$ gas-stripped tail. In the central few arcseconds of the galaxy, we report a possible marginal detection of about $1 \times 10^{6} M_{\odot}$ of molecular gas (assuming a Galactic CO-to- $\mathrm{H}_{2}$ conversion factor) that could correspond to a surviving nuclear gas reservoir. We estimate that there is less molecular gas in the main body of IC 3418, by at least a factor of 20 , than would be expected from the pre-quenching UV-based star formation rate assuming the typical gas depletion timescale of $2 \mathrm{Gyr}$. Given the lack of star formation in the main body, we think the $\mathrm{H}_{2}$-deficiency is real, although some of it may also arise from a higher $\mathrm{CO}$-to- $\mathrm{H}_{2}$ factor typical in low-metallicity, low-mass galaxies. The presence of $\mathrm{H}$ II regions in the tail of IC 3418 suggests that there must be some dense gas; however, only upper limits of $<1 \times 10^{6} M_{\odot}$ were found in the three observed points in the outer tail. This yields an upper limit on the molecular gas content of the whole tail $<1 \times 10^{7} M_{\odot}$, which is an amount similar to the estimates from the observed star formation rate over the tail. We also present strong upper limits on the X-ray emission of the stripped gas in IC 3418 from a new Chandra observation. The measured X-ray luminosity of the IC 3418 tail is about 280 times lower than that of ESO 137-001, a spiral galaxy in a more distant cluster with a prominent ram pressure stripped tail. Non-detection of any diffuse X-ray emission in the IC 3418 tail may be due to a low gas content in the tail associated with its advanced evolutionary state and/or due to a rather low thermal pressure of the surrounding intra-cluster medium.
\end{abstract}

Key words. galaxies: individual: VCC 1217/IC 3418 - galaxies: clusters: individual: Virgo - galaxies: evolution - galaxies: ISM ISM: kinematics and dynamics - methods: observational

\section{Introduction}

The evolution of galaxies in clusters is driven by interactions between the interstellar medium (ISM) of the galaxies and the gas in the intra-cluster medium (ICM; Gunn \& Gott 1972; Poggianti et al. 1999; van Gorkom 2004; Koopmann \& Kenney 2004). Because of its proximity and dynamical youth (Schindler 1999; Randall et al. 2008), the Virgo Cluster is an excellent laboratory for studying the processes that transform galaxies in dense environments. By far the most numerous galaxies in Virgo are the dwarfs. The relationship between the blue star-forming dwarf irregulars (dIs) and the more common dwarf ellipticals (dEs) has been a long-standing puzzle. Dwarf ellipticals are centrally concentrated in the cluster, whereas blue star-forming dwarf irregulars are preferentially located in the outskirts, although their masses and structural parameters are similar (Binggeli et al. 1987). It has therefore been suggested that dEs might transform

* Based on observations carried out with the IRAM 30 m Telescope and with the Chandra X-ray Observatory. IRAM is supported by INSU/CNRS (France), MPG (Germany), and IGN (Spain).

$\star \star$ CO spectra as FITS files are available at the CDS via anonymous ftp to cdsarc.u-strasbg. fr $(130.79 .128 .5)$ or via http://cdsarc.u-strasbg.fr/viz-bin/qcat?]/A+A/556/A99 from gas-rich dwarf galaxies which lose their interstellar gas and subsequently quench their star formation because of ram pressure stripping (Boselli et al. 2008). Objections to this scenario based on differences in kinematics, metallicity, and nuclei in the $\mathrm{dI}$ and $\mathrm{dE}$ populations have been largely convincingly addressed by Kormendy \& Bender (2012). But until recently there has not been any clear case of a galaxy actually undergoing such a transformation.

The galaxy IC 3418 (VCC 1217) is a peculiar dwarf galaxy located very close to the cluster core, with a projected distance of only $1^{\circ}(\sim 290 \mathrm{kpc})$ from M87 (see Fig. 1), and a line-of-sight velocity with respect to the cluster mean of about $-1000 \mathrm{~km} \mathrm{~s}^{-1}$. It is very likely on its first orbit through the Virgo center, and probably close to its nearest approach to M87. No H I was detected anywhere in the galaxy down to a $3 \sigma$ limit of $\sim 8 \times 10^{6} M_{\odot}$ per beam (Chung et al. 2009); in the main body of the galaxy there is also no $\mathrm{H} \alpha$ emission (Hester et al. 2010). The stellar metallicity of the galaxy was measured from optical spectroscopy to be $\sim 0.5 \pm 0.2 Z_{\odot}$ (Kenney et al., in prep.). A new stellar population analysis indicates that star formation in the galaxy stopped about $250 \pm 50 \mathrm{Myr}$ ago in the central $25^{\prime \prime}$, and that it took $\$ 70$ Myr for star formation to cease from $\sim 40^{\prime \prime}$ to the center (Kenney et al., in prep.). This is consistent with ram 
Table 1. Parameters of IC 3418 (VCC 1217).

\begin{tabular}{|c|c|}
\hline RA, Dec (J2000) & $12: 29: 43.8,+11: 24: 09$ \\
\hline Type & $\mathrm{IBm}$ \\
\hline$v_{\text {helio }}{ }^{a}$ & $170 \pm 10 \mathrm{~km} \mathrm{~s}^{-1}$ \\
\hline$D_{\mathrm{M} 87}$ projected & $1.02^{\circ}(\approx 290 \mathrm{kpc})^{h}$ \\
\hline Major/minor diameter ${ }^{b}$ & $1.5^{\prime} / 1^{\prime}(\approx 7.2 \mathrm{kpc} / 4.8 \mathrm{kpc})^{h}$ \\
\hline $\mathrm{PA}$, inclination ${ }^{c}$ & $\sim 45^{\circ}, \sim 50^{\circ}$ \\
\hline Apparent/absolute $B$ mag. ${ }^{d}$ & $14.85 /-16.2$ \\
\hline Apparent/absolute $K$ mag. ${ }^{e}$ & $12.55 /-18.5$ \\
\hline FUV, NUV, FUV-NUV ${ }^{f}$ & $17.55,16.69,0.85 \pm 0.10$ \\
\hline H I mass ${ }^{g}$ & $<8 \times 10^{6} M_{\odot}(3 \sigma)$ \\
\hline H I-deficiency ${ }^{g}$ & $>2.16$ \\
\hline Tail projected length & $3.5^{\prime}(\approx 17 \mathrm{kpc})^{h}$ \\
\hline
\end{tabular}

References. (a) Kenney et al. (in prep.); $38 \mathrm{~km} \mathrm{~s}^{-1}$ in GoldMine (Gavazzi et al. 2003); ${ }^{(b)}$ NED ; ${ }^{(c)}$ HyperLEDA (Paturel et al. 2003); (d) Internal and Galactic extinction corrected (Gavazzi et al. 2006). Corresponding $B$-band luminosity is $4.8 \times 10^{8} L_{\odot}$, using $M_{B, \odot}=5.48$ (e.g., Mo et al. 2010); ${ }^{(e)}$ GoldMine. Foreground Galactic extinction in the $K$-band is 0.012 mag (NED). Corresponding $K$-band luminosity is $5.2 \times 10^{8} L_{\odot}$, using $M_{K, \odot}=3.28$ (e.g., Mo et al. 2010); ${ }^{(f)}$ Gil de Paz et al. (2007); ${ }^{(g)}$ Chung et al. (2009); ${ }^{(h)}$ Assuming Virgo cluster distance of $16.5 \mathrm{Mpc}$ (Mei et al. 2007).

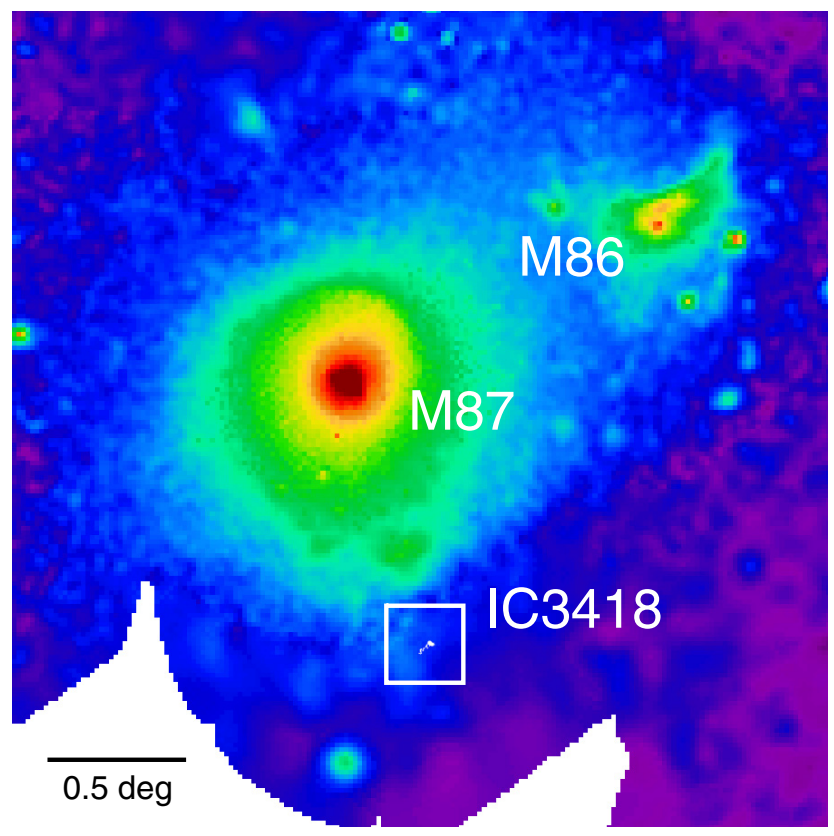

Fig. 1. Location of IC 3418 in the Virgo cluster. The background image shows the distribution of the $0.5-2.0 \mathrm{keV}$ ICM as observed in X-rays with ROSAT (Credit: S. L. Snowden, http: //heasarc.gsfc.nasa. gov). Silhouette of a GALEX UV image of IC 3418 is displayed at the position of the galaxy.

pressure suppression of star formation from the outside inwards, acting typically on a timescale of $\sim 100$ Myr. The main stellar body of the galaxy is symmetric and undisturbed indicating that a tidal interaction is excluded (Kenney et al., in prep.).

A ram pressure stripping scenario for IC 3418 is also suggested by optical and GALEX UV observations (Chung et al. 2009; Hester et al. 2010; Fumagalli et al. 2011) that revealed a remarkable one-sided tail extending $17 \mathrm{kpc}$ from the galaxy's SE side. It comprises about nine bright knots and linear, parallel filaments of young UV-bright stars. Only in the outer half of the tail $\mathrm{H} \alpha+[\mathrm{N}$ II $]$ emission is found, mostly associated with

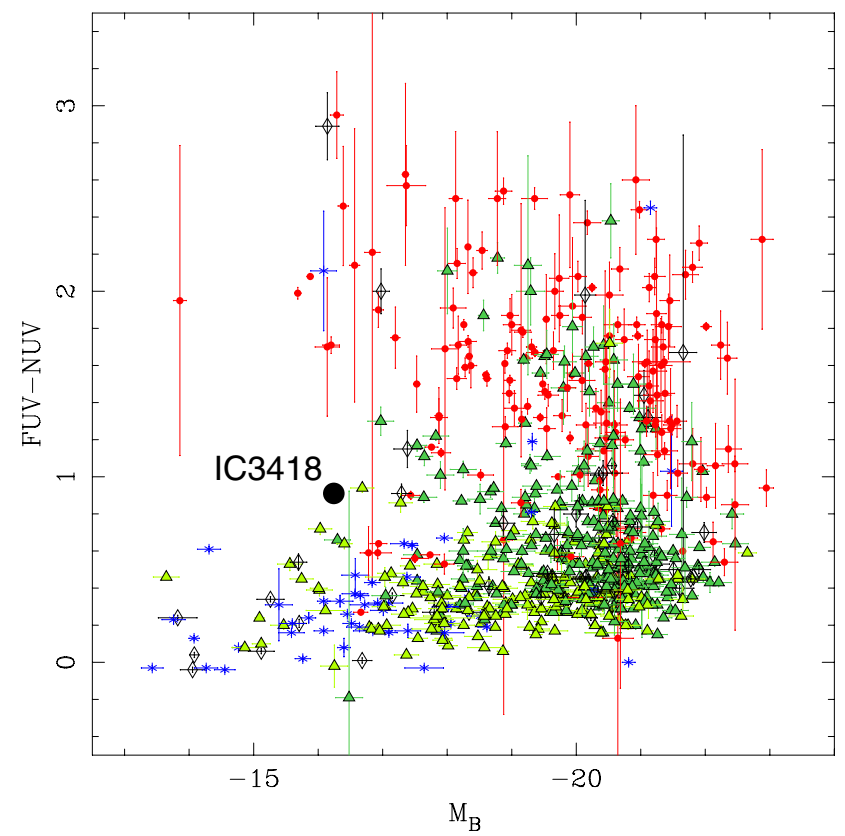

Fig. 2. Location of IC 3418 in the (FUV - NUV) $-M_{B}$ color-magnitude diagram of nearby galaxies from the sample of Gil de Paz et al. (2007). FUV - NUV $=0.85 \pm 0.10$ (Gil de Paz et al. 2007) and $m_{B}$ corrected for internal and Galactic extinction is $14.85 \mathrm{mag}$ (NED), i.e., $M_{B}=-16.2$. Red dots are elliptical/lenticular galaxies, dark green triangles are earlytype spirals, light green triangles are late-type spirals, blue asterisks are irregular and compact galaxies, and black diamonds are galaxies lacking morphological classification. Figure adapted from Gil de Paz et al. (2007)

fireballs (Yoshida et al. 2008), elongated streams of young stars with $\mathrm{H}$ II regions and bright UV knots at their heads (Kenney et al., in prep.). These are interpreted as dense, star-forming gas clouds which are accelerated by ram pressure, leaving behind trails of newly-formed stars that are not affected by ram pressure and therefore decouple from the gas.

While good examples of ram pressure stripping are known among the Virgo spiral galaxies (e.g., Kenney et al. 2004; Chung et al. 2007, 2009; Abramson et al. 2011), none of these galaxies is completely gas stripped and none has such a luminous UV tail, or linear parallel stellar streams, or fireballs as IC 3418. There are already known examples of one-sided tails of young stars extending from more massive galaxies in more distant, richer clusters (Cortese et al. 2007; Yoshida et al. 2008; Smith et al. 2010; Sun et al. 2010; Woudt et al. 2008), where the ram pressure can be 1 to 2 orders of magnitude stronger than in Virgo, but IC 3418 is by far the closest such galaxy known.

In Fig. 2, IC 3418 is placed into a (FUV - NUV) vs. $M_{B}$ color-magnitude diagram of a sample of nearby galaxies studied by Gil de Paz et al. (2007). It shows that IC 3418 stands outside both the red and blue sequences of galaxies, respectively, and that it occurs right at the boundary between early- and latetype galaxies. This suggests that IC 3418 is being transformed from a dwarf irregular type to an early-type galaxy (see Kenney et al., in prep.) which is consistent with the recent cease of its star formation due to ram pressure stripping.

Numerical modeling has shown that ram pressure stripping is a very efficient process for removing the gaseous content of cluster galaxies (e.g., Vollmer et al. 2001; Roediger \& Hensler 2005; Jáchym et al. 2007, 2009). Simulations also predict that material in the gas-stripped tails gets compressed by ram pressure and radiative cooling can form new molecular clouds in situ, which 


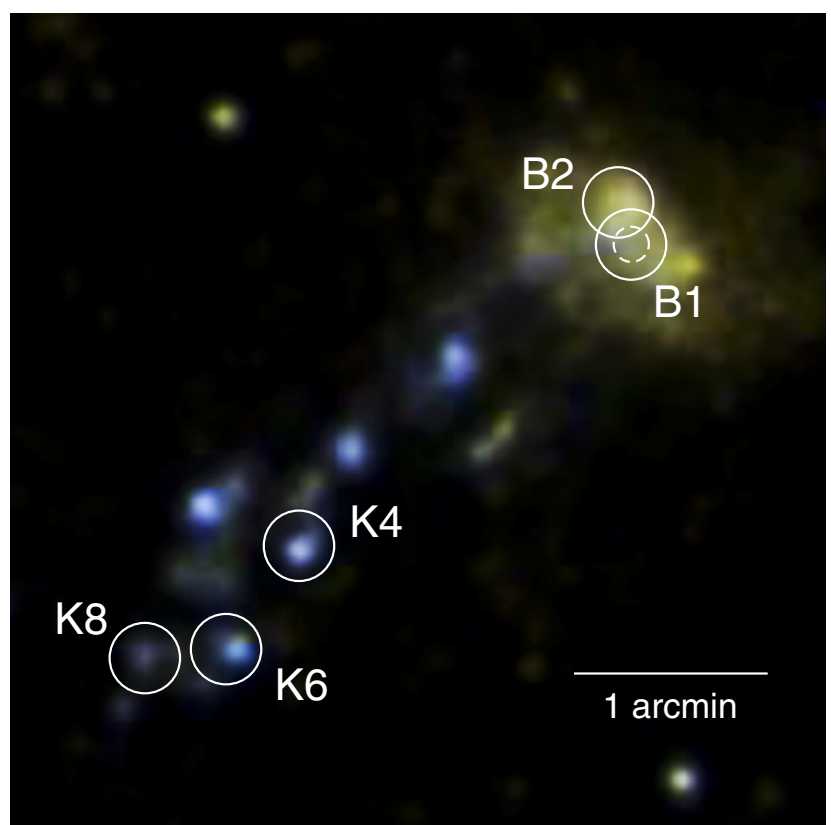

Fig. 3. Observed positions on a GALEX image (FUV in blue, NUV in yellow) of IC 3418 with ${ }^{12} \mathrm{CO}(1-0)$ beams $\left(F W H M=21^{\prime \prime}\right)$ of the $30 \mathrm{~m}$ IRAM telescope displayed - two positions (B1, B2) in the main body of the galaxy and three $(\mathrm{K} 4, \mathrm{~K} 6, \mathrm{~K} 8)$ in the outer part of the tail. The $\mathrm{CO}(2-1)$ beams have the same centers but half the diameter as the circles shown - a $\mathrm{CO}(2-1)$ beam is shown in $\mathrm{B} 1$ with a dashed circle. The background image is adopted from Hester et al. (2010).

might then form a population of new stars (Kapferer et al. 2009; Tonnesen \& Bryan 2009). Dwarf galaxies have weaker potential wells and their ambient ISM may be correspondingly less dense (Bolatto et al. 2008). This, plus a strong ram pressure near the cluster center, could significantly enhance stripping of IC 3418 and thus account for its peculiar morphology. The presence of star formation in the tail, strongly suggesting the presence of molecular gas, makes IC 3418 an attractive place to search for $\mathrm{CO}$ emission in the environmentally affected galaxy. However, dwarf irregulars are usually fainter in $\mathrm{CO}$ than large spirals when normalized by (stellar) mass and those with metallicities less than $\sim 8.0$ (in oxygen abundance scale) are challenging to detect (e.g., Taylor et al. 1998; Komugi et al. 2011).

In this paper we want to address two main questions: (1) Is the main body of IC 3418 totally stripped or did some gas survive the stripping? We present the results of our IRAM $30 \mathrm{~m}$ search for $\mathrm{CO}$ emission since molecular gas is more likely to survive than H I. (2) How does the ISM behave in ram pressure stripped, star-forming tails? We are interested in searching for molecular and for hot X-ray emitting gas in the tail in order to help characterize the ISM in such tails. We present the results of our new sensitive Chandra observation of the galaxy.

The structure of the paper is as follows: after a brief description of our observations (Sect. 2), we present and analyze our IRAM 30 m results (Sect. 3), and estimate upper limits on the current molecular gas content of the whole IC 3418 (Sect. 4). We then estimate the original molecular gas content of the galaxy from the star formation rate (SFR) in the main body and compare the molecular content of IC 3418 with other low-mass galaxies (Sect. 5). The $\mathrm{H}_{2}$-deficiency of the galaxy is also discussed. In Sect. 6 we analyze the Chandra results. Then, by means of numerical calculations we study the effects of ram pressure on ISM with different column density (Sect. 7). In Sect. 8 we discuss the structure of the gas-stripped tail, as well as the fate of the
Table 2. List of observed positions.

\begin{tabular}{cccccc}
\hline \hline & $\begin{array}{c}\text { RA } \\
(\mathrm{J} 2000)\end{array}$ & $\begin{array}{c}\text { Dec } \\
(\mathrm{J} 2000)\end{array}$ & $\begin{array}{c}T_{\mathrm{ON}} \\
(\mathrm{hr})\end{array}$ & $\begin{array}{c}T_{\text {sys }}^{(1-0)} \\
(\mathrm{K})\end{array}$ & $\begin{array}{c}T_{\text {sys }}^{(2-1)} \\
(\mathrm{K})\end{array}$ \\
\hline B1 & $12: 29: 43.8$ & $+11: 24: 09$ & 5.6 & $240-300$ & $400-500$ \\
B2 & $12: 29: 44.07$ & $+11: 24: 22.38$ & 2.8 & $200-300$ & $230-400$ \\
K4 & $12: 29: 50.75$ & $+11: 22: 36.35$ & 2.5 & $250-350$ & $400-700$ \\
K6 & $12: 29: 52.29$ & $+11: 22: 04.86$ & 2.7 & $250-330$ & $500-600$ \\
K8 & $12: 29: 53.92$ & $+11: 22: 01.85$ & 1.7 & $210-280$ & $240-310$ \\
\hline
\end{tabular}

Notes. System temperatures are given in $T_{\mathrm{A}}^{*}$ scale.

stripped gas, and origin of star formation in the tail. The conclusion follows in Sect. 9.

\section{Observations}

\subsection{Millimeter integration: IRAM $30 \mathrm{~m}$}

Using the $30 \mathrm{~m}$ telescope operated by the Institut de Radio Astronomie Millimétrique (IRAM) at Pico Veleta, Spain, in August 2011 we carried out observations towards the main body of IC 3418 and the brightest $\mathrm{H} \alpha / \mathrm{UV}$ regions of its tail (see scheme in Fig. 3). We used the EMIR receiver in E090 and E230 bands to observe simultaneously at the frequencies of the ${ }^{12} \mathrm{CO}(1-0)\left(v_{\text {rest }}=115.271 \mathrm{GHz}\right)$ and the ${ }^{12} \mathrm{CO}(2-1)$ $\left(v_{\text {rest }}=230.538 \mathrm{GHz}\right)$ lines. At these frequencies the telescope half-power beamwidths are $21^{\prime \prime}$ and $11^{\prime \prime}$, respectively, which corresponds to a resolution of about $1.7 \mathrm{kpc}$ and $0.9 \mathrm{kpc}$, respectively, at the adopted distance of the Virgo cluster of $16.5 \mathrm{Mpc}$ (Mei et al. 2007). As backends, we used the $4 \mathrm{MHz}$ filterbanks at $115 \mathrm{GHz}$ with $10.4 \mathrm{~km} \mathrm{~s}^{-1}$ velocity resolution, and the WILMA autocorrelator with a spectral resolution of $2 \mathrm{MHz}$ at both $115 \mathrm{GHz}$ and $230 \mathrm{GHz}$ (i.e., $5.2 \mathrm{~km} \mathrm{~s}^{-1}$ velocity resolution at $115 \mathrm{GHz}$ and $2.6 \mathrm{~km} \mathrm{~s}^{-1}$ at $230 \mathrm{GHz}$ ). The new FTS spectrometer with $0.192 \mathrm{kHz}$ spectral resolution was also connected to both lines as a back-up. In this work, WILMA and 4MHz data are analyzed.

All observations were done in the Wobbler switching mode that is known to provide much flatter baselines, with ripples at a much lower amplitude than with the position switching mode. The secondary reflector was switching symmetrically at $0.7 \mathrm{kHz}$ frequency with $120^{\prime \prime}$ amplitude in azimuth. The actual distance between the source and a reference position is double the amplitude value, i.e., $240^{\prime \prime}$, which is large enough to just avoid the tail with the standard azimuthal direction of switching.

The main beam efficiencies $B_{\text {eff }}$ of the $30 \mathrm{~m}$ antenna are estimated to 0.79 at $115 \mathrm{GHz}$ and 0.57 at $230 \mathrm{GHz}$, and the forward efficiencies $F_{\text {eff }}$ to 0.94 and 0.92 , respectively. The corrected antenna temperatures provided by the telescope were converted to the main beam brightness temperature by $T_{\mathrm{mb}}=T_{\mathrm{A}}^{*} F_{\text {eff }} / B_{\text {eff }}$. To check the system setup we observed IRC+10216 and M99 and we obtained lines matching the catalogued lines.

Weather conditions were typical for the summer afternoon period at Pico Veleta with the PWV exceeding $10 \mathrm{~mm}$ in the first half of the run. Then, after a passage of a weather front, the conditions improved substantially and PWV decreased to 5-7 mm. The atmosphere was, however, rather unstable and pointing and focusing were difficult. Pointing was checked about every $1.5 \mathrm{~h}$ mostly on Saturn which was close to our sources, usually with small corrections from $2^{\prime \prime}$ to $5^{\prime \prime}$. The system temperatures are given in Table 2 . 
Table 3. Properties of the observed positions in IC 3418.

\begin{tabular}{|c|c|c|c|c|c|c|c|c|}
\hline Source & $\begin{array}{c}\mathrm{rms}_{\mathrm{W}(1-0) / \mathrm{W}(2-1) / 4 \mathrm{MHz}} \\
(\mathrm{mK}) \\
(2)\end{array}$ & $\begin{array}{c}F W H M \\
\left(\mathrm{~km} \mathrm{~s}^{-1}\right) \\
(3)\end{array}$ & $\begin{array}{c}I_{\mathrm{CO}(1-0)} \\
\left(\mathrm{K} \mathrm{km} \mathrm{s}^{-1}\right) \\
(4)\end{array}$ & $\begin{array}{c}I_{\mathrm{CO}(2-1)} \\
\left(\mathrm{K} \mathrm{km} \mathrm{s}^{-1}\right) \\
(5)\end{array}$ & $\begin{array}{c}L_{\mathrm{CO}(1-0)} \\
\left(10^{5} \mathrm{~K} \mathrm{~km} \mathrm{~s}^{-1} \mathrm{pc}^{2}\right) \\
(6)\end{array}$ & $\begin{array}{c}L_{\mathrm{CO}(2-1)} \\
\left(10^{5} \mathrm{~K} \mathrm{~km} \mathrm{~s}^{-1} \mathrm{pc}^{2}\right) \\
(7)\end{array}$ & $\begin{array}{l}M_{\mathrm{mol}}^{\mathrm{CO}(1-0)} \\
\left(10^{6} M_{\odot}\right) \\
\quad(8)\end{array}$ & $\begin{array}{c}M_{\mathrm{mol}}^{\mathrm{CO}(2-1)} \\
\left(10^{6} M_{\odot}\right) \\
\quad(9)\end{array}$ \\
\hline B1 & $1.1 / 1.6 / 1.0$ & $54 \pm 12$ & $0.06 \pm 0.03$ & $0.24 \pm 0.05$ & 2.1 & 2.1 & 0.9 & 1.2 \\
\hline B2 & $1.4 / 2.0 / 1.3$ & $\sim 30$ & $<0.1$ & $<0.1$ & $<2.7$ & $<0.9$ & $<1.2$ & $<0.5$ \\
\hline K4 & $2.0 / 3.6 / 1.8$ & $\sim 30$ & $<0.1$ & $<0.2$ & $<3.8$ & $<1.7$ & $<1.7$ & $<0.9$ \\
\hline K6 & $2.0 / 4.0 / 1.6$ & $\sim 30$ & $<0.1$ & $<0.2$ & $<3.7$ & $<1.9$ & $<1.6$ & $<1.0$ \\
\hline K8 & $1.7 / 2.3 / 1.7$ & $\sim 30$ & $<0.1$ & $<0.1$ & $<3.2$ & $<1.1$ & $<1.4$ & $<0.6$ \\
\hline
\end{tabular}

Notes. The central velocity of the line in the B1 position is $103 \mathrm{~km} \mathrm{~s}^{-1}$. Column(1) source name. Column (2) noise levels in WILMA CO(1-0), WILMA CO(2-1), and $4 \mathrm{MHZ}$ spectra with $10.4 \mathrm{~km} \mathrm{~s}^{-1}$ channels. From fitting first-order baselines in a $2000 \mathrm{~km} \mathrm{~s}^{-1}$ wide region of the spectra. Column (3) linewidths used for both $\mathrm{CO}(1-0)$ and $\mathrm{CO}(2-1)$ lines. Measured in $\mathrm{B} 1$ from the $\mathrm{CO}(2-1)$ detection, estimated for other positions (see the text). Column (4) detected (B1) or $3 \sigma$ upper limits on WILMA CO(1-0) integrated intensity. Column (5) detected (B1) or $3 \sigma$ upper limits on $\mathrm{CO}(2-1)$ integrated intensity. Column (6) detected (B1) or $3 \sigma$ upper limits on $\mathrm{CO}(1-0)$ luminosity. Column (7) detected (B1) or $3 \sigma$ upper limits on $\mathrm{CO}(2-1)$ luminosity. Column (8) detected (B1) or $3 \sigma$ upper limits on molecular gas mass, including a factor of 1.36 to account for the effect of He, corresponding to WILMA CO(1-0) luminosity. Column (9) detected (B1) or $3 \sigma$ upper limits on molecular gas mass, including a factor of 1.36 to account for the effect of He, corresponding to WILMA CO(2-1) luminosity.

The sky coordinates of the observed positions shown in Fig. 3 are given in Table 2, together with respective on-source times. These were typically $2.5 \mathrm{~h}$ per position per polarization, divided into 6 min scans. For the B1 position it was roughly double. For K8, the on-source time was shorter by a factor of $\sim 1.5$ than for the $\mathrm{K} 4$ and $\mathrm{K} 6$, but observing conditions had improved resulting in a better sensitivity. The data were reduced with CLASS (Continuum and Line Analysis Single-dish Software) developed by IRAM in the standard manner: the spectra were checked for errors (spikes) and bad channels exceeding the $5 \sigma$ level were flagged. The quality of WILMA and $4 \mathrm{MHz}$ data was very good. Linear baselines were fitted. Each spectrum was re-binned to a velocity resolution of $10.4 \mathrm{~km} \mathrm{~s}^{-1}$ and both polarizations were averaged in order to increase the signal-tonoise ratio $(\mathrm{S} / \mathrm{N})$. All scans were then summed up to produce the final spectra. The root mean square (rms) noise levels of about $1-2 \mathrm{mK}$ at $\mathrm{CO}(1-0)$ frequency were achieved in the final summed spectra (Table 3).

\section{2. $X$-ray imaging: Chandra}

The observation of IC 3418 was performed with the Advanced CCD Imaging Spectrometer (ACIS) on November 12, 2012 (obsID: 13811). Standard Chandra data analysis was performed which includes the corrections for the slow gain change, charge transfer inefficiency, and the ACIS low-energy quantum efficiency degradation from the contamination on the ACIS optical blocking filter. No flares of particle background were present in the observation. The effective exposure time is $33.8 \mathrm{ks}$ for the S3 chip where IC 3418 is positioned. CIAO4.4 was used for the data analysis. The calibration files used correspond to Chandra calibration database 4.5.3 from the Chandra X-ray Center. The solar photospheric abundance table by Anders \& Grevesse (1989) were used in the spectral fits. We adopted an absorption column density of $2.2 \times 10^{20} \mathrm{~cm}^{-2}$ from the Leiden/Argentine/Bonn H I survey (Kalberla et al. 2005).

\section{Results - IRAM}

In a set of deep integrations we observed two positions in the main body of the galaxy where dense molecular gas might have survived the effects of the cluster environment, and three positions in the outer tail where the presence of dense gas is suggested from the existence of $\mathrm{H}$ II regions (Fig. 3, see coordinates in Table 2). The B1 pointing is centered at the galaxy's optical center (as given by NED), and the B2 point is shifted by less than one $\mathrm{CO}(1-0)$ beamwidth in the NE direction, towards the brightest region of the galaxy where significant substructure occurs, indicating that star formation occurred there recently. The tail positions $\mathrm{K} 4, \mathrm{~K} 6$, and $\mathrm{K} 8^{1}$ are associated with peaks in UV and $\mathrm{H} \alpha$ emission.

\subsection{Main body}

First we searched for $\mathrm{CO}$ emission in the central position B1. This was our deepest integration with the total on-source time of about $5.6 \mathrm{~h}$ (per polarization). The left panels in Fig. 4 show WILMA $\mathrm{CO}(2-1)$ and WILMA $\mathrm{CO}(1-0)$ spectra smoothed to $10.4 \mathrm{~km} \mathrm{~s}^{-1}$ resolution. Very low rms values of about $1.7 \mathrm{mK}$ and $1.1 \mathrm{mK}$, respectively, were achieved. Although no strong detection appears, a feature that is six channels wide $\left(\sim 60 \mathrm{~km} \mathrm{~s}^{-1}\right)$ occurs at both frequencies at the central velocity of about $100 \mathrm{~km} \mathrm{~s}^{-1}$. It is more prominent in the $\mathrm{CO}(2-1)$ spectrum. We inspected the surrounding parts of the spectra and found no other similar case. The $\mathrm{S} / \mathrm{Ns}$ of the line feature in the WILMA $\mathrm{CO}(1-0)$ and WILMA $\mathrm{CO}(2-1)$ spectra are 2.1 and 5.6, respectively, where we have calculated $S / N=N^{-1 / 2} \sigma_{\text {rms }}^{-1} \sum_{i=1}^{N} I_{i}$, where $N$ is the number of channels covered by the line, $\sigma_{\mathrm{rms}}$ is the rms intensity of the spectrum per channel, and $I_{i}$ is the brightness temperature in the $i$ th channel. In order to claim a detection, we require the integrated intensity $I_{\mathrm{CO}}=\int T_{\mathrm{mb}} \mathrm{d} v$ to be at least three times greater than the noise over the spectral channels covered by the line ${ }^{2}$.

There is no statistically significant line detection in the $\mathrm{CO}(1-0)$ WILMA and $4 \mathrm{MHz}$ spectra of the central position, but there are 2.0-2.7- $\sigma$ features with the same velocity range as the $\mathrm{CO}(2-1)$ line. The integrated $\mathrm{CO}(1-0)$ intensity measured from the WILMA spectrum is $\sim 0.06 \mathrm{~K} \mathrm{~km} \mathrm{~s}^{-1}$ (see Table 3 ). The $\mathrm{CO}(1-0)$ and $\mathrm{CO}(2-1)$ features are consistent with a compact $\mathrm{CO}$ source which suffers much more beam dilution in the $21^{\prime \prime}$ $\mathrm{CO}(1-0)$ beam than the $11^{\prime \prime} \mathrm{CO}(2-1)$ beam. With four times

1 The naming of the tail positions corresponds to Hester et al. (2010). Fumagalli et al. (2011) use a different notation (K-6, K-4, K-3).

2 The noise over $N$ spectral channels covered by the line $\sigma_{\mathrm{I}}=$ $\Delta v_{\text {res }} \sigma_{\text {rms }} N^{1 / 2}=\sigma_{\text {rms }}\left(\Delta v_{\text {res }}\left\langle\Delta v_{\text {CO }}\right\rangle\right)^{1 / 2}$, where $\Delta v_{\text {res }}$ is the velocity resolution and $\left\langle\Delta v_{\mathrm{CO}}\right\rangle$ is the mean FWHM linewidth. 

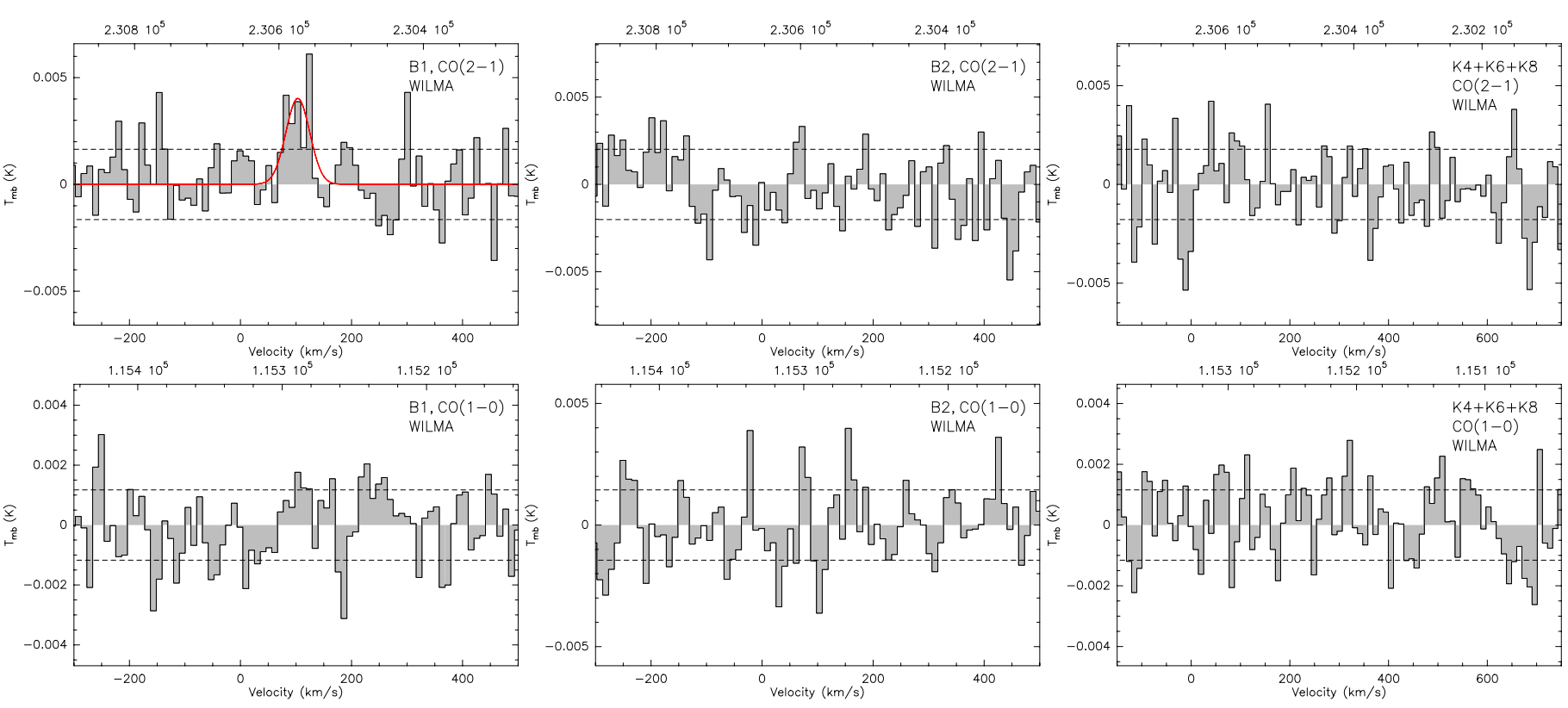

Fig. 4. Marginal detection in the central main body position B1 (left), spectra in the off-center position B2 (middle), and combined K4+K6+K8 tail spectra (right): WILMA CO(2-1) (top) and WILMA CO(1-0) (bottom) spectra smoothed to $10.4 \mathrm{~km} \mathrm{~s}^{-1}$ resolution. Dashed lines show $\pm 1 \sigma$ noise levels; $y$-axis scaling is set to $\pm 4 \sigma$. The velocity scale is LSR; for the sky position of IC $3418, v_{\text {helio }}=v_{\text {LSR }}-3.2 \mathrm{~km} \mathrm{~s}^{-1}$.

the beam area, a point-like source will experience four times the beam dilution in the $\mathrm{CO}(1-0)$ beam. The ratio of peak temperatures of $\sim 3$ and integrated intensities of $\sim 4$ are consistent with the factor of 4 expected from maximum beam dilution multiplied by the typical $I_{\mathrm{CO}(2-1)} / I_{\mathrm{CO}(1-0)}$ line ratio of $0.8-1$ in molecular clouds (e.g., Leroy et al. 2008).

We thus consider the $\mathrm{CO}(2-1)$ emission in the center of IC 3418 as marginally detected. In Fig. 4, the $\mathrm{CO}(2-1)$ spectral line is fitted with a Gaussian profile with the central velocity of $103 \mathrm{~km} \mathrm{~s}^{-1}$, FWHM of $54 \mathrm{~km} \mathrm{~s}^{-1}$, peak brightness temperature of $4.3 \mathrm{mK}$, and the integrated intensity $I_{\mathrm{CO}(2-1)}=$ $0.24 \pm 0.05 \mathrm{~K} \mathrm{~km} \mathrm{~s}^{-1}$. The central velocity of the line is somewhat smaller than that deduced by Kenney et al. (in prep.) from the stellar Keck spectra (see Table 1). We discuss the velocity difference in Sect. 8.1. Since there is no HI emission in IC 3418, we estimate the maximum rotation velocity from the Tully-Fisher relation (by comparing the $H$-band luminosity of IC 3418 to other Virgo galaxies with known rotation velocities) to be about $55-60 \mathrm{~km} \mathrm{~s}^{-1}$. Correcting for an inclination of $\sim 50^{\circ}$ (Chung et al. 2009), the expected linewidth is about $50 \mathrm{~km} \mathrm{~s}^{-1}$, similar to the observed linewidth.

At the adopted Virgo distance of $16.5 \mathrm{Mpc}$, the main beam projected area at $\mathrm{CO}(2-1)$ frequency is $\Omega_{B} \simeq 137 \operatorname{arcsec}^{2}=$ $0.9 \mathrm{kpc}^{2}$, including a Gaussian beamshape correction factor of $1 / \ln 2$. The B1 integrated intensity then corresponds to a luminosity $L_{\mathrm{CO}(2-1)}=2.1 \times 10^{5} \mathrm{~K} \mathrm{~km} \mathrm{~s}^{-1} \mathrm{pc}^{2}$. The corresponding molecular gas mass can be calculated using $M_{\mathrm{H}_{2}}\left[M_{\odot}\right]=$ $5.5 X_{\mathrm{CO}} \frac{R_{21}}{0.8} L_{\mathrm{CO}}\left[\mathrm{K} \mathrm{km} \mathrm{s}^{-1} \mathrm{pc}^{2}\right]$, where $X_{\mathrm{CO}}=N_{\mathrm{H}_{2}} / I_{\mathrm{CO}}$ is the $\mathrm{CO}(1-0)$-to- $\mathrm{H}_{2}$ conversion factor normalized to a standard Galactic value of $2 \times 10^{20} \mathrm{~cm}^{-2}\left(\mathrm{~K} \mathrm{~km} \mathrm{~s}^{-1}\right)^{-1}$ (e.g., Kennicutt \& Evans 2012; Pineda et al. 2010; Feldmann et al. 2012), and $R_{21}$ is the $\mathrm{CO}(2-1)$ to $\mathrm{CO}(1-0)$ ratio. We assume a typical value of $R_{21}=0.8$ (e.g., Leroy et al. 2008). The above formula includes a factor of 1.36 to account for the effects of helium. The resulting amount of molecular gas possibly detected in the B1 main body position is $1.2 \times 10^{6} M_{\odot}$. In Sect. 5.2 we discuss that the $\mathrm{X}$-factor may be (somewhat) larger than the standard Galactic value because of the sub-solar metallicity of IC 3418 .
The size of the emitting region in the $\mathrm{B} 1$ pointing is certainly comparable to or smaller than the $\mathrm{CO}(2-1)$ beam, i.e., $\$ 900 \mathrm{pc}$. This is most likely gas concentrated in the nuclear region. A lower limit on the column density of the central CO-emitting blob is $\propto M_{\mathrm{mol}} / \Omega_{B} \sim 1 M_{\odot} \mathrm{pc}^{-2}$, assuming a standard Galactic conversion X-factor, if gas uniformly fills the beam. It is, however, much more likely to be concentrated over a smaller area. The actual gas surface density could thus be similar to that of a typical giant molecular cloud (GMC), or $\sim 100 M_{\odot} \mathrm{pc}^{-2}$. Also the B1 luminosity of $2.1 \times 10^{5} \mathrm{~K} \mathrm{~km} \mathrm{~s}^{-1} \mathrm{pc}^{2}$ is comparable to the luminosity of a single giant Galactic molecular cloud. However, the linewidth is much larger than that from a single GMC with $M_{\text {gas }} \simeq 10^{6} M_{\odot}$ (typically $\sim 10 \mathrm{~km} \mathrm{~s}^{-1}$ ) which strongly suggests that emission is not from a single GMC.

In the off-center main body position B2 (about $15^{\prime \prime} \sim 1.2 \mathrm{kpc}$ from B1) covering the NE young stellar complex (which is the brightest optical part of the galaxy), no $\mathrm{CO}$ emission was detected (see Fig. 4, middle panels). This suggests that molecular gas has possibly survived the effects of the cluster environment only in the very center of the galaxy. In the B2 position, we assume an upper limit on the line width to be about $30 \mathrm{~km} \mathrm{~s}^{-1}$, i.e., smaller than at B1, as it does not cover the rotation center. Rotation curve studies of Local Volume dwarfs (e.g., Kirby et al. 2012) show that at $r=1 \mathrm{kpc}$, the rotation velocity is $0.5 v_{\max } \sim 30 \mathrm{~km} \mathrm{~s}^{-1}$. Thus, we derive the upper limits on the integrated CO intensity, CO luminosity, and molecular gas mass, given in Table 3.

\subsection{Stripped tail and fireballs}

We searched for $\mathrm{CO}$ emission in the three points $\mathrm{K} 4$, K6, and $\mathrm{K} 8$ covering most of the bright $\mathrm{H}$ II regions in the outer tail (see Fig. 3). The positions K4 and K6 show a head-tail (fireball) structure with $\mathrm{H} \alpha$ offset from the UV peaks. We did not observe the brightest UV region (K5 in the notation of Hester et al. 2010) and instead went for the more distant knot K8 that has a higher $\mathrm{H} \alpha / \mathrm{UV}$ ratio than $\mathrm{K} 5$, suggesting that it may be at an earlier evolutionary stage with more gas. The galaxy IC 3418 is moving 

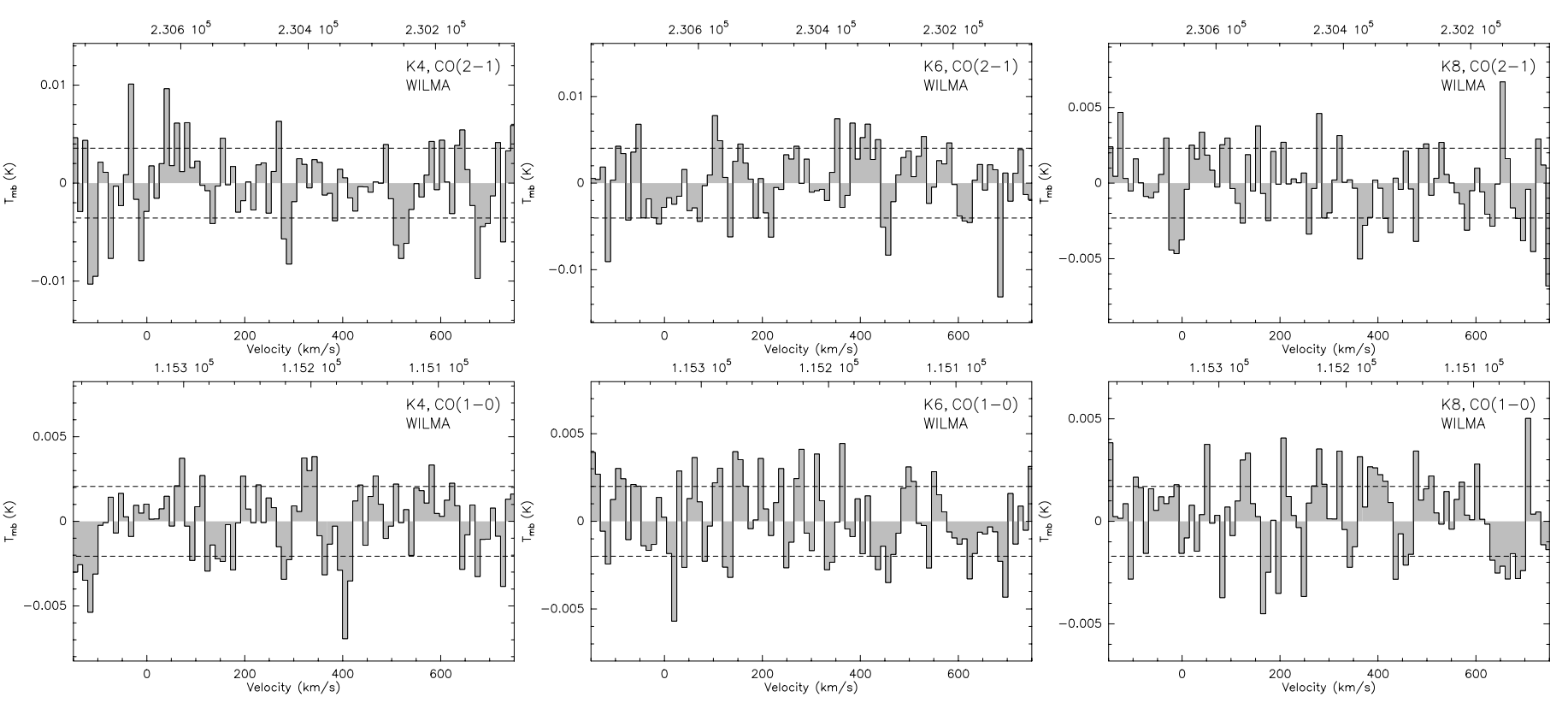

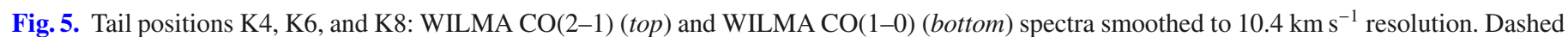
lines show $\pm 1 \sigma$ noise levels; $y$-axis scaling is set to $\pm 4 \sigma$.

toward us with respect to M87 and the stripped material is thus extending away from us: we expect it to be accelerated to larger line-of-sight velocities.

In Fig. 5 we inspect the WILMA spectra for the K4, K6, and $\mathrm{K} 8$ regions over a large range, from velocities of the main body to those several hundred $\mathrm{km} \mathrm{s}^{-1}$ higher. Although there are few $\sim 3 \sigma$ features resembling spectral lines, they never occur in the spectra at both frequencies simultaneously, as in the case of B1. As we will show later in Figs. 9 and 10, only low-column density ISM parcels could be accelerated by ram pressure to large vertical velocities. No clear line emission is thus detected in any of the three tail positions. Since the tail linewidths might be broadened by galactic rotation, we will assume linewidths of $\sim 30 \mathrm{~km} \mathrm{~s}^{-1}$ to derive upper limits on $\mathrm{CO}$ intensity in all three tail positions. Table 3 gives corresponding upper limits on $I_{\mathrm{CO}}$, $L_{\mathrm{CO}}$, and $M_{\mathrm{mol}}$. The upper limits on the molecular gas mass are $\sim 1 \times 10^{6} M_{\odot}$ per point, assuming a standard Galactic X-factor, but it is possible that in the $\mathrm{H} \alpha$ bright tail regions the CO-to- $\mathrm{H}_{2}$ conversion factor is different from standard value (see discussion in Sect. 5.2).

In order to increase the SNR, we also combined data from the three positions, assuming for a moment that conditions of the molecular gas are similar and that their radial velocities are close; however, no significant signal occurred in the stacked spectrum (see the right panels in Fig. 4). Our achieved upper limits per point on CO luminosity are comparable to the luminosity across most of the SMC, $L_{\mathrm{SMC}, \mathrm{CO} 10} \sim 1 \times 10^{5} \mathrm{~K} \mathrm{~km} \mathrm{~s}^{-1} \mathrm{pc}^{2}$ as measured by Mizuno et al. (2001). With $\mathrm{CO}(1-0)$ point luminosities of $\sim 2 \times 10^{5} \mathrm{~K} \mathrm{~km} \mathrm{~s}^{-1} \mathrm{pc}^{2}$, we are sensitive enough to detect analogues to the brightest clouds in M33 or the LMC N197 complex.

\section{Current molecular gas content}

In this section we will estimate from our IRAM observations upper limits on the molecular content in the whole galaxy. We will also calculate an upper limit on the molecular gas mass following from dust observations of IC 3418.
Table 4. Molecular gas mass estimates corresponding to our IRAM detection and upper limits on $\mathrm{CO}$ luminosity in the main body and in the tail, assuming a Galactic CO-to- $\mathrm{H}_{2}$ conversion factor.

\begin{tabular}{lcc}
\hline \hline Source & $\begin{array}{c}L_{\mathrm{CO}(2-1)} \\
\left(10^{5} \mathrm{~K} \mathrm{~km} \mathrm{~s}^{-1} \mathrm{pc}^{2}\right)\end{array}$ & $\begin{array}{c}M_{\text {mol }} \\
\left(10^{6} M_{\odot}\right)\end{array}$ \\
\hline Center (B1) & 2.1 & 1.2 \\
Total disk & $\geq 2.1,<9$ & $\geq 1.2,<5$ \\
Total tail & $<18$ & $<10$ \\
Body + tail & $<27$ & $<15$ \\
\hline
\end{tabular}

\subsection{Current upper limits - the whole galaxy}

In Table 4 we give estimates (upper limits) of the CO luminosity and molecular gas mass in the central beam of the main body (the B1 position), the entire main body, the tail, and the whole galaxy (main body plus tail). To estimate the total CO luminosity of the main body of the galaxy from the measured B1 and B2 (upper limit) luminosities, we estimate the filling factor of the $\mathrm{CO}(2-1)$ and $\mathrm{CO}(1-0)$ beams as the ratio of their area to the optical surface of the galaxy, assuming an exponential distribution of $\mathrm{CO}$ emission with a scale length roughly 1.5 times smaller than the optical scale length (Young et al. 1995). Fumagalli et al. (2011) fitted the surface brightness of the IC 3418 disk with an exponential law with a scale length of $19^{\prime \prime} \simeq 1.5 \mathrm{kpc}$. Accounting for the $R_{25}$ major axis diameter of $\sim 7.2 \mathrm{kpc}$ and the inclination of $\sim 50^{\circ}$ (see Table 1), we estimate that the $\mathrm{CO}(1-0)$ main beam located in the B1 position would encompass about $25 \%$ of the total disk CO luminosity. This yields an estimated upper limit on the molecular gas content in the whole galaxy of about $5 \times 10^{6} M_{\odot}$. Leroy et al. (2008) suggested that typical dwarfs in a sample of nearby galaxies have molecular gas distributed mostly in their inner $\left(<0.25 r_{25}\right)$ radius, which matches the $\mathrm{CO}(1-0)$ beam radius. Moreover, since the galaxy is probably gas-stripped from the outside in, the expected gas distribution would be more compact than the stellar disk. Thus, it is likely that we are detecting a larger fraction of the total CO flux in the central beam. 
Applying our $3 \sigma$ upper limit of $M_{\text {mol }} \lesssim 1 \times 10^{6} M_{\odot}$ measured in each of the three tail regions (K4, K6, and K8) to the total number of knots occurring in the wake, an upper limit on the total molecular mass in the tail would be $\sim 9 \times 10^{6} M_{\odot}$. A similar value comes from calculating the filling factor of the three $\mathrm{CO}(1-0)$ beams in the whole tail area. Assuming that the molecular gas occurs (mostly) in the actual places of star formation, and taking into account that all $\mathrm{H}$ II regions are observed only in the outer half of the tail, the upper limit could possibly be lowered to a value similar to the one derived above for the main body. Nevertheless, we conclude that the upper limit on the current molecular gas content of the whole galaxy (main body plus tail) is about $1.5 \times 10^{7} M_{\odot}$.

\subsection{Current molecular gas amount from dust emission}

Far infrared (FIR) dust measurements allow an alternative approach for estimating the current molecular gas mass that overcomes some disadvantages of traditional $\mathrm{CO}$ line tracers. It traces the total gas column density $\left(\mathrm{HI}+\mathrm{H}_{2}\right)$. In the Small Magellanic Cloud, IRAS observations suggested much more $\mathrm{H}_{2}$ than seen from CO (Israel 1997; Leroy et al. 2007a) and, thus, that $\mathrm{CO}$ may be underabundant or absent in regions where $\mathrm{H}_{2}$ survives because the $\mathrm{H}_{2}$ self-shields while the $\mathrm{CO}$ is photodissociated (e.g., Maloney \& Black 1988).

From ISO observations Tuffs et al. (2002) obtained $3 \sigma$ upper limits on $60 \mu \mathrm{m}, 100 \mu \mathrm{m}$, and $170 \mu \mathrm{m}$ FIR dust emission of IC 3418 of $<0.04 \mathrm{Jy},<0.03 \mathrm{Jy}$, and $<0.07 \mathrm{Jy}$, respectively. The total dust mass can be determined to within a factor of 3 from (e.g., Boselli et al. 2002; Evans et al. 2005)

$M_{\text {dust }}=C S_{100 \mu \mathrm{m}} D^{2}\left(\mathrm{e}^{a / T_{\text {dust }}}-1\right)$,

where $C$ relates to the grain opacity, $S_{100 \mu \mathrm{m}}$ is the flux in the $100 \mu \mathrm{m}$ band in Jy, $D$ is the distance of the galaxy in Mpc, and $T_{\text {dust }}$ is the dust temperature. According to Boselli et al. (2002), we use $C=1.27 M_{\odot} \mathrm{Jy}^{-1} \mathrm{Mpc}^{-2}, T_{\text {dust }}=20.8 \mathrm{~K}$, and $a=144 \mathrm{~K}$ for $100 \mu \mathrm{m}$ emission. For the $S_{100 \mu \mathrm{m}}<0.03$ Jy upper limit we get a limit on the dust mass of $\lesssim 10^{4} M_{\odot}$. Taking a typical cold gas-to-dust ratio of about 500 (e.g., Lisenfeld \& Ferrara 1998), we obtain an upper limit on the molecular gas mass in the galaxy of $\lesssim 5 \times 10^{6} M_{\odot}$. This is a value similar to our CO luminositybased upper limit estimate (see Table 4). As we will discuss in the following section, it is likely that molecular gas has been effectively stripped from IC 3418. This would imply that some dust also has been removed from the galaxy by ram pressure stripping.

\section{Original molecular gas content}

We estimate the molecular gas mass $M_{\text {mol }}$ likely to have been in IC 3418 before it was ram pressure stripped from the SFR in the main body. The far-ultraviolet (FUV) emission is a tracer of star formation in the last couple 100 Myr. The FUV luminosity within the $25 \mathrm{mag} \operatorname{arcsec}^{-2} B$-band isophote of the main body of IC 3418 (corrected for Galactic extinction) is $L_{\mathrm{FUV}}=1.4 \times 10^{41} \mathrm{erg} \mathrm{s}^{-1}$ (Gil de Paz et al. 2007). The classical SFR-luminosity relation works well for continuous star formation approximation where SFR is constant over the timescale of the UV emission. This is probably not true in IC 3418, where star formation ceased about $200 \mathrm{Myr}$ ago as a result of ram pressure stripping of star-forming ISM, and so the current FUV luminosity is likely scaled down because the stellar population had faded. From evolutionary stellar population modeling we can estimate that FUV flux in a case where star formation ended $100 \mathrm{Myr}$ or $200 \mathrm{Myr}$ ago is reduced by a factor of 8 or 20 , respectively, compared to a model with continuous star formation (Hugh Crowl, priv. comm.). This factor could be somewhat lower if ram pressure induced a burst of star formation before quenching it, a phenomenon that was identified in the outer radii of NGC 4522 (Crowl \& Kenney 2006), for example. For IC 3418 this is suggested from measuring equivalent widths of the absorption Balmer lines in the spectrum of IC 3418, which are stronger than in post star-burst $\mathrm{k}+\mathrm{a}$ galaxies (Fumagalli et al. 2011).

As a result, we will apply a factor of $\sim 10$ to the SFR of $6.1 \times 10^{-3} M_{\odot} \mathrm{yr}^{-1}$ determined from the FUV luminosity-SFR formula of Kennicutt \& Evans (2012)

$\log \operatorname{SFR}\left(M_{\odot} \mathrm{yr}^{-1}\right)=\log L_{\mathrm{FUV}}\left(\mathrm{erg} \mathrm{s}^{-1}\right)-43.35$.

To account for sub-solar metallicities appropriate for dwarf galaxies, we will also apply to the SFR the factor of $1 / 1.1$ estimated by Hunter et al. (2010) from the stellar population evolution models of STARBURST99 (Leitherer et al. 1999) for luminosity at $1500 \AA$. This then yields an original SFR of $\sim 5.6 \times 10^{-2} M_{\odot} \mathrm{yr}^{-1}$ in IC 3418 , a value that is consistent with measurements in other dwarf galaxies where SFRs can span several orders of magnitude (e.g., Hunter et al. 2010; Schruba et al. 2012). Assuming a typical star formation efficiency in dwarf galaxies of $\tau_{\text {dep }} \approx 2$ Gyr (e.g., Bigiel et al. 2008), this yields about $1 \times 10^{8} M_{\odot}$ of molecular gas that originally was in the galaxy. This is about 20 times more than our CO-based upper limit estimate of the current molecular gas content of the main body.

Another rough estimate of the original molecular amount can come from the stellar mass of the galaxy and typical fractions of atomic and molecular gas seen in galaxies of the same type. However, this estimate is affected by the issue of the $\mathrm{X}$-factor that we will deal with later. Bothwell et al. (2009) calculated the stellar mass-to-light ratios for different galaxy morphology-color combinations using the algorithm of Bell \& de Jong (2001). For Im and dIrr types they derived $M / L_{B}=0.49$. For the $B$-band magnitude $m_{B}=14.85$ (see Table 1 ) of IC 3418 this yields a stellar mass $M_{*} \approx 3 \times 10^{8} M_{\odot}$, which is consistent with the estimate of Fumagalli et al. $(2011)\left(\sim 3.8 \times 10^{8} M_{\odot}\right)$. It is known that ISMs of dwarf galaxies are dominated by large reservoirs of atomic gas - along the Hubble sequence the H I fraction increases up to typically $M_{\mathrm{HI}} / M_{*}>0.5$ or even $>1$ in the late types (e.g., Bell \& de Jong 2000). The original H I mass of IC 3418 is estimated to $\sim 6 \times 10^{8} M_{\odot}$ (Gavazzi et al. 2005), which is in agreement with the expected $M_{\mathrm{HI}} / M_{*}>1$. Although dwarf galaxies are HI-rich, molecular gas represents only a small fraction of their total gas mass, typically $M_{\mathrm{H}_{2}} / M_{\mathrm{H}_{\mathrm{I}}} \approx 0.1-0.2$ which is similar to the ratio found in the outer parts of spiral galaxies (e.g., Obreschkow \& Rawlings 2009; Israel 1997). This corresponds to $\sim(0.6-1) \times 10^{8} M_{\odot}$ of original $\mathrm{H}_{2}$ in IC 3418 , which is consistent with the above estimate.

\subsection{Comparison with other galaxies}

We will also compare our results with other galaxies, including low-mass, low-metallicity dwarfs. In Fig. 6, IC 3418 is placed into a plot of normalized CO luminosity $L_{\mathrm{CO}} / L_{K}$ vs. $L_{K}$, together with (a) the sample of Schruba et al. (2012) containing 16 nearby low-mass star-forming HERACLES galaxies as well as some more massive HERACLES galaxies and Local Group galaxies, (b) a subset of the sample of compact late-type spirals 


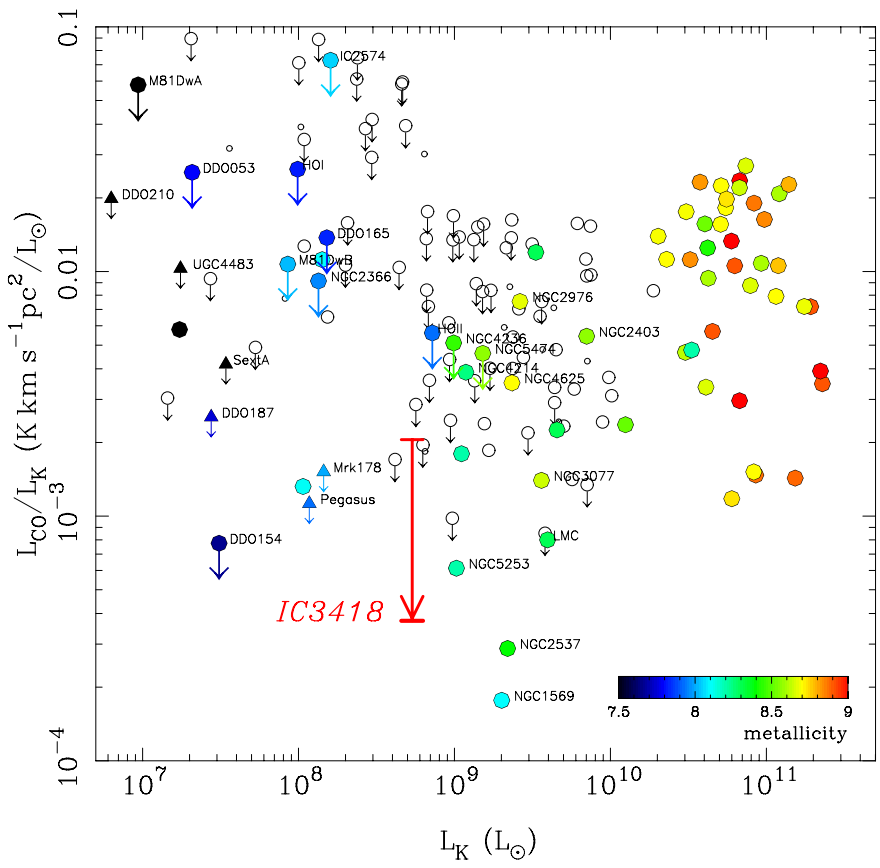

Fig. 6. Normalized $\mathrm{CO}(1-0)$ luminosity as a function of $K$-band luminosity. IC 3418 is represented with a red arrow with the head corresponding to the marginally detected (B1) CO luminosity and the upper tail bar to the upper limit for the total luminosity of the disk. IC 3418 is compared with a set of nearby low-mass star-forming galaxies from the HERACLES survey (together with more massive HERACLES galaxies, and other Local Group and nearby galaxies) used by Schruba et al. (2012) (filled circles), and with a sample of compact late-type spiral and irregular galaxies of Leroy et al. (2005) (empty circles), plus few starburst dwarf galaxies from Taylor et al. (1998) (triangles), and DDO154 (Komugi et al. 2011). Upper limits are plotted with symbols with small arrows. Colors represent metallicity $12+\log (O / H)$. The color of the IC 3418 arrow does not correspond to its metallicity (see Sect. 5.2). Small empty circles show marginal detections of Leroy et al. (2005).

and irregulars of Leroy et al. (2005, their detections correspond instead to lower limits because they observed only the central few kpc of each galaxy), (c) a few starburst dwarfs of Taylor et al. (1998) not contained in the Schruba et al. (2012) collection, and (d) DDO154 observed by Komugi et al. (2011).

The normalization $L_{\mathrm{CO}} / L_{K}$ used in Fig. 6 should remove most of the galactic mass and size correlations and thus reveal an unbiased state of the $\mathrm{CO}$ content of the galaxies. All the $K$-band magnitudes of the galaxies were taken from NED, as well as their distances (except for the sample of Schruba et al. 2012, for which we used their distances). We preferred to use redshift-independent (mostly Tully-Fisher) distances whenever available. The $K$-band apparent magnitude of IC 3418 is 12.55 (see Table 1). The $\mathrm{CO}(2-1)$ luminosity detected in the $\mathrm{B} 1$ position was converted to $\mathrm{CO}(1-0)$ scale using $I_{\mathrm{CO} 21}=0.7 I_{\mathrm{CO} 10}$, to be consistent with Schruba et al. (2012).

In Fig. 6 IC 3418 is represented with a red arrow with the head corresponding to the detected (B1) CO luminosity and the upper tail bar to the upper limit for the total luminosity of the disk. When first focusing on the high end of the IC 3418 range, the galaxy occurs in the middle of other galaxies and thus shows no special value of $L_{\mathrm{CO}} / L_{K}$. However, when taking into account only the actual detection in the nuclear region of the disk, IC 3418 shifts to a rather sparsely populated region of the plot where only few galaxies have similar or lower values of the normalized $\mathrm{CO}$ luminosities. Although this region may suffer from a lack of observations with CO sensitivity levels similar to ours, this suggests that $\mathrm{CO}$ content of IC 3418 is low relative to its other properties. The $L_{\mathrm{CO}} / L_{K}$ ratio is systematically lower in galaxies with $L_{K} \lesssim 10^{10} L_{\odot}$, probably mostly because of a different $\mathrm{CO}-$ to- $\mathrm{H}_{2}$ relation connected to their low metallicity. But even among low-mass galaxies of a given metallicity, the CO-to- $\mathrm{H}_{2}$ relationship may vary due to variations in the amount of photo-dissociating UV radiation, which is correlated with the SFR. The other dwarfs with similar or lower values of $L_{\mathrm{CO}} / L_{K}$, notably NGC 1569, NGC 2537, or NGC 5253, are all starburst blue compact dwarfs in which strong photo-dissociation of molecular gas by UV radiation from young stars may make their CO content especially low. In IC 3418, since star formation ended about 100-200 Myr ago, the effects of photo-dissociation are expected to be less extreme.

Thus, the reason for the low $L_{\mathrm{CO}} / L_{K}$ ratio of IC 3418 must be different. Since the galaxy clearly has been strongly ram pressure stripped, the removal of molecular or molecular-forming ISM would be a possible explanation.

\section{2. $\mathrm{CO}$ as a tracer of $\mathrm{H}_{2}$}

It has been shown that in dwarf irregular galaxies with metallicities lower than about $1 / 10$ of solar, i.e., $12+\log (\mathrm{O} / \mathrm{H}) \leq 8.0$, the detection of CO emission steeply decreases (e.g., Taylor et al. 1998; Leroy et al. 2007b; Komugi et al. 2011; Schruba et al. 2012), although ionized carbon and the ratio of IR to millimeter dust emission around star-forming regions indicate that molecular gas is still present. Applying a Galactic CO-to- $\mathrm{H}_{2}$ conversion ratio then results in very low molecular gas masses and about 10-100 times higher star formation efficiencies (SFEs). It is, however, more likely that the true SFEs of dwarfs are normal, and the CO-to- $\mathrm{H}_{2}$ factor is systematically different. Thus, assuming a constant $\mathrm{H}_{2}$ depletion time of $\tau_{\text {dep }}=1.8 \mathrm{Gyr}$, Schruba et al. (2012) derived for dwarf galaxies with metallicities $1 / 2-1 / 10 Z_{\odot}$ conversion factors more than one order of magnitude larger than in massive spirals with solar metallicities.

Using evolutionary models for Lick indices in Keck spectroscopic data, stellar metallicity that represents an average over the star formation history in IC 3418 is estimated to $0.5 \pm 0.2$ solar, i.e., $12+\log (\mathrm{O} / \mathrm{H}) \sim 8.4$ (Kenney et al., in prep. $)^{3}$. Another estimate comes from the stellar mass-metallicity relation (Tremonti et al. 2004; Kewley \& Ellison 2008; Pettini \& Pagel 2004): for the IC 3418 stellar mass of $\sim 3 \times 10^{8} M_{\odot}$, the predicted stellar metallicity is $12+\log (\mathrm{O} / \mathrm{H}) \sim 8.3$, i.e., $\sim 0.4$ solar. Unfortunately, there is no data available on the gas metallicity of IC 3418 that would reveal the actual amount of metals currently present in the ISM. Nevertheless, in IC 3418 we expect the CO-to- $\mathrm{H}_{2}$ conversion factor to be larger than Galactic because of the decreased dust shielding at a presumably low metallicity in the galaxy. From Eq. (5) of Leroy et al. $(2013)^{4}$ the correction to the standard CO-to- $\mathrm{H}_{2}$ conversion factor can be estimated to $\sim 5$. However, this estimate remains highly uncertain.

\section{3. $\mathrm{H}_{2}$-deficiency?}

While many Virgo cluster spirals are highly H I-deficient (e.g., Kenney et al. 2004; Chung et al. 2007, 2009; Abramson et al. 2011), their molecular gas content is relatively normal or only 3 We assume a solar value of $12+\log (\mathrm{O} / \mathrm{H})=8.69$ (Asplund et al.
2009 ).
$4 \quad c_{\text {CO,dark }} \approx 0.65 \exp \left[0.4 /(D / G)^{\prime}\right]$, where $(D / G)^{\prime}$ is the dust-to-gas surface density of a GMC of $100 M_{\odot} \mathrm{pc}^{-2}$ is adopted. 
slightly deficient (e.g., Kenney \& Young 1986, 1989). This is largely a difference between the inner $\mathrm{H}_{2}$-dominated, and outer H I-dominated disks. In galaxies which are clearly ram pressure stripped, nearly all the gas, including molecular gas, and dust beyond some gas truncation radius is gone (Vollmer et al. 2012; Cortese et al. 2010). While decoupled molecular clouds have been observed beyond the main gas truncation radius in some galaxies (NGC 4402, NGC 4522, NGC 4438; Crowl et al. 2005; Vollmer et al. 2008; Vollmer 2009, respectively), the mass in such features is small and they apparently do not survive long. Thus, ram pressure stripped spirals in Virgo must be somewhat $\mathrm{H}_{2}$-deficient ${ }^{5}$.

The IC 3418 galaxy is highly HI-deficient, with the deficiency parameter $\operatorname{def}_{\mathrm{HI}}=\log \left(M_{\mathrm{H} \text { I,orig }} / M_{\mathrm{HI}, \mathrm{obs}}\right) \gtrsim 2.16$ (Chung et al. 2009; Gavazzi et al. 2005). The above estimated original amount of molecular gas in the galaxy of $\sim 1 \times 10^{8} M_{\odot}$ in comparison with our CO-based upper limit of $5 \times 10^{6} M_{\odot}$ indicates that IC 3418 could also be poorer in molecular gas by a factor of 20 (i.e., $\left.\operatorname{def}_{\mathrm{H}_{2}}=\log \left(M_{\mathrm{H}_{2} \text {,orig }} / M_{\mathrm{H}_{2} \text {,obs }}\right) \sim 1.3\right)$. The $\mathrm{H}_{2}$-deficiency is generally small in Virgo spirals since ram pressure is not strong enough to strip massive galaxies deeply. However, lower mass galaxies with shallower potential wells are expected to be more completely stripped in Virgo, and are expected to be significantly $\mathrm{H}_{2}$-deficient; IC 3418 may be such a galaxy. While we cannot presently determine how much of the weakness in $\mathrm{CO}$ emission is due to $\mathrm{H}_{2}$-deficiency vs. a non-standard CO-to- $\mathrm{H}_{2}$ relation, the lack of ongoing star formation in IC 3418 strongly suggests the galaxy is deficient in molecular gas.

\section{Results - Chandra}

No diffuse X-ray emission is detected from IC 3418 and its tail. There is also no detection of a shock front ahead of IC 3418. The Chandra data show three point sources in the tail region, which will be discussed elsewhere. In this paper, we focus on the limit of the X-ray emitting gas in the tail region $\left(3.5^{\prime} \times 1^{\prime}\right.$, or $16.8 \mathrm{kpc} \times 4.8 \mathrm{kpc})$. A $3 \sigma$ upper limit on the X-ray enhancement over the Virgo ICM background emission at the tail region is derived from the $0.5-2 \mathrm{keV}$ image. A spectral model of the X-ray tail is required to convert the count rate limit to the flux limit and the mass limit. The X-ray tail is expected to have multiple temperature components. However, low statistics of X-ray tails so far have hindered a detailed study, so often a single temperature component is assumed (see detailed discussions in Sun et al. 2010 ). The known X-ray tails have $T_{\mathrm{ICM}} / T_{\text {tail }}$ ratios of 3-8 (Sun \& Vikhlinin 2005; Sun et al. 2010; Weżgowiec et al. 2011). If we simply take this range for the ratio, the expected tail temperature of IC 3418 is $0.3-0.8 \mathrm{keV}$, for a surrounding ICM temperature of $\sim 2.5 \mathrm{keV}$ (Shibata et al. 2001). In this work, we assume a single APEC model with temperatures of $0.3-0.7 \mathrm{keV}$ and abundances of 0.1 solar or 1 solar (see Table 5). The assumed X-ray tail is approximated by a cylinder $16.8 \mathrm{kpc}$ long with a diameter of $4.8 \mathrm{kpc}$. The derived upper limits are listed in Table 5. While it is often assumed that the stripped ISM is metal rich, a one- $T$ fit always results in very low abundance, which is presumably caused by the mixing of multi- $T$ gas in the tail (see discussions in Sun et al. 2010).

\footnotetext{
5 Fumagalli et al. (2009) have recently reported $\mathrm{H}_{2}$-deficient galaxies, although these are anemic galaxies, with low surface densities of H I and $\mathrm{H}_{2}$ out to large radii. Whereas truncated galaxies are found almost exclusively in clusters and are clearly ram pressure stripped, the origin of the anemic galaxies, which are found both inside and outside of clusters, is unclear, although they may be starved galaxies.
}

Table 5. Upper limits on the X-ray emission from the IC 3418 tail.

\begin{tabular}{ccccc}
\hline \hline $\begin{array}{c}\text { Model } T / Z \\
\text { (keV/solar) }\end{array}$ & $\begin{array}{c}L_{0.5-2} \\
\left(10^{38} \mathrm{erg} / \mathrm{s}\right)\end{array}$ & $\begin{array}{c}L_{\text {bol }} \\
\left(10^{38} \mathrm{erg} / \mathrm{s}\right)\end{array}$ & $\begin{array}{c}n_{\mathrm{e}} f_{\mathrm{X}}^{-1 / 2} \\
\left(10^{-3} \mathrm{~cm}^{-3}\right)\end{array}$ & $\begin{array}{c}M_{\mathrm{X}} f_{\mathrm{X}}^{1 / 2} \\
\left(10^{7} M_{\odot}\right)\end{array}$ \\
\hline $0.3 / 0.1$ & 3.5 & 13 & 4.3 & 3.7 \\
$0.3 / 1.0$ & 3.5 & 7.8 & 1.6 & 1.4 \\
$0.5 / 0.1$ & 3.1 & 7.2 & 3.1 & 2.7 \\
$0.5 / 1.0$ & 3.0 & 4.5 & 1.2 & 1.1 \\
$0.7 / 0.1$ & 2.9 & 6.1 & 2.7 & 2.4 \\
$0.7 / 1.0$ & 2.7 & 4.0 & 1.1 & 0.97 \\
\hline
\end{tabular}

Notes. $L_{0.5-2}$ : rest-frame $0.5-2 \mathrm{keV}$ luminosity. $L_{\mathrm{bol}}$ : X-ray bolometric luminosity. $n_{\mathrm{e}}$ : electron density. $f_{\mathrm{X}}$ : X-ray volume filling factor. $M_{\mathrm{X}}$ : $\mathrm{X}$-ray mass.

As shown in Table 5, the derived $0.5-2 \mathrm{keV}$ luminosity limit is $\sim 280$ times and $\sim 100$ times lower than the luminosities of ESO 137-001 and ESO 137-002, respectively, the spiral galaxies in a more distant cluster with prominent ram pressure stripped tails with strong X-ray emission (Sun et al. 2010). The X-ray mass limit is less than $3.7 \times 10^{7} f_{\mathrm{X}}^{1 / 2} M_{\odot}$, where $f_{\mathrm{X}}$ is the filling factor of the X-ray emitting gas in the tail. If the X-ray tail in IC 3418 is cooler than the $0.3 \mathrm{keV}$ assumed in our spectral model, the mass and bolometric luminosity limits would become weaker.

In numerical simulations the amount of X-ray emitting gas in the tail was found to depend strongly on the surrounding ICM pressure (Tonnesen et al. 2011). The thermal pressure of the ICM at the location of IC 3418 in Virgo is $P_{\mathrm{ICM}}=n_{\mathrm{ICM}} T \sim$ $2.8 \times 10^{-12}$ dyne $\mathrm{cm}^{-2}$, where $n_{\mathrm{ICM}}=7.1 \times 10^{-4} \mathrm{~cm}^{-3}$ is the local ICM density (see Sect. 7 for details), and $k_{B} T=2.5 \mathrm{keV}$ is the local ICM temperature (Shibata et al. 2001). The simulation run of Tonnesen \& Bryan (2010) with a close ICM thermal pressure value of $1.76 \times 10^{-12} \mathrm{dyne}^{-2}$ suggests that some soft $(0.5-2 \mathrm{keV}) \mathrm{X}$-ray emission could be observable in the tail of IC 3418 for our sensitive Chandra surface brightness limit of $\sim 4 \times 10^{-7} \mathrm{erg} \mathrm{s}^{-1} \mathrm{~cm}^{-2}$. This follows from Fig. 2 (right panel) in Tonnesen et al. (2011), assuming the same column density of about $10^{20} \mathrm{~cm}^{-2}$ for the hot gas in the tail of IC 3418 as is measured in the simulations. However, the gas mass and gas density in the tail are expected to strongly evolve over time, and Tonnesen \& Bryan (2010) did not study the evolutionary stage after the galaxy is fully stripped, like in IC 3418.

Once the main body of the galaxy has been (nearly) completely stripped, the source of new tail gas is depleted. In one of the simulations of Kapferer et al. (2009), who use a different numerical hydrodynamics scheme than does Tonnesen (smoothed particle hydrodynamics, SPH, vs. adaptive mesh refinement, AMR), a model massive galaxy is completely stripped by a strong ram pressure. Some $400 \mathrm{Myr}$ after the galaxy was stripped, there is still a very long bright X-ray tail that is spatially coincident with a star-forming tail. However, IC 3418 is a small galaxy, originally with a much smaller amount of gas. Thus, after the main body has been nearly completely stripped, the gas density of the tail is expected to drop. It is possible that the X-ray tail lags farther behind the SF tail which is the case in ESO 137-001, for example, where the brightest X-ray region is $35 \mathrm{kpc}$ from the galaxy. However, the Chandra field of view covers another $\sim 2.2^{\prime}$ after the end of the SF tail, with no sign of the X-ray enhancement. The X-ray surface brightness is about the same, so the flux limit would be $\sim 28 \%$ higher if a region of $5.7^{\prime} \times 1^{\prime}$ is considered (instead of $\left.3.5^{\prime} \times 1^{\prime}\right)$. 
Table 6. Parameters of galaxy and cluster models used for semi-analytic calculations of ram pressure stripping.

\begin{tabular}{clc}
\hline \hline Galaxy: & $M_{\mathrm{d}}=5 \times 10^{8} M_{\odot}$ & $a_{\mathrm{d}}=1.5 \mathrm{kpc}$ \\
& $M_{\mathrm{h}}=7 \times 10^{9} M_{\odot}$ & $a_{\mathrm{h}}=3 \mathrm{kpc}$ \\
\hline Cluster: & $M_{\text {vir }}=1.4 \times 10^{14} M_{\odot}$ & $r_{\mathrm{s}}=320 \mathrm{kpc}$ \\
& $\delta_{\text {th }}=340$ & $\Omega_{\mathrm{m}}=0.3$ \\
& $\rho_{\text {ICM }, 1}(0)=4.4 \times 10^{-22} \mathrm{~kg} \mathrm{~m}^{-3}$ & $r_{c, 1}=1.2 \mathrm{kpc}$ \\
& $\rho_{\text {ICM }, 2}(0)=1.4 \times 10^{-23} \mathrm{~kg} \mathrm{~m}^{-3}$ & $r_{c, 2}=23.7 \mathrm{kpc}$ \\
& $\beta_{1}=0.42$ & $\beta_{2}=0.52$ \\
\hline
\end{tabular}

Thus, one reason for the weak X-ray (as well as $\mathrm{H} \alpha$ and $\mathrm{HI}$ ) emission in the tail of IC 3418 could be that it is in an advanced evolutionary stage when the gas density of the tail is expected to drop. In the Virgo cluster, however, almost no X-ray tails are observed in other ram pressure stripped galaxies with $\mathrm{HI}$ or $\mathrm{H} \alpha$ tails. The possible reasons for our non-detection thus further include low pressure of the surrounding ICM (for comparison, the ICM thermal pressure at the position of ESO 137-001 in the A3627 cluster is about $1.8 \times 10^{-11}$ dyne $\mathrm{cm}^{-2}$, i.e., about six times higher than in Virgo at the position of IC 3418) or a lower tail temperature than we assumed in the spectral model, which would also mean fast cooling (assuming a not-very-small abundance) and thus a short lifetime.

\section{Ram pressure stripping}

Ram pressure stripping is very likely the principal cause of the strong gas deficiency of IC 3418 and of its peculiar morphology (Hester et al. 2010; Fumagalli et al. 2011). Acceleration due to ram pressure basically depends on the column density of the gas parcels and the gravitational potential of the galaxy. The shallow potential of the dwarf makes its interstellar matter more susceptible to ram pressure and so material with higher column density can be affected. Compared to other ram pressure stripped galaxies in the Virgo cluster, IC 3418 is also projected closer to the cluster center where the ICM density is higher. Although we don't know the true de-projected distance, IC 3418 is probably affected by a stronger ram pressure than other Virgo galaxies.

In this section we use semi-analytic calculations to model the effects of a time-varying ram pressure on both atomic and molecular ISM components in a simple manner by taking into account their column density as a critical parameter. In a static potential of a two-component model of the galaxy consisting of the disk and halo (the contribution of a bulge is neglected), we follow dynamics of ISM parcels at different disk radii under the influence of an external time-varying face-on force. Every parcel is assigned a column density. For every parcel we calculate the equation of motion in the vertical direction that takes into account local ram pressure and the restoring force from the galaxy. The local ram pressure may be expressed as $\mathrm{d} v / \mathrm{d} t=$ $-\rho_{\text {ICM }}\left|v-v_{0}\right|^{2} / \Sigma_{\text {ISM }}$, where $\Sigma_{\text {ISM }}$ is the mean column density of an ISM parcel and $\left(v-v_{0}\right)$ is the vertical component of its relative velocity with respect to the surrounding hot gas. The galaxy is modeled with a Miyamoto-Nagai disk and a Plummer halo (e.g., Binney \& Tremaine 2008) with the parameters specified in Table 6.

At the projected distance of IC 3418 from M87 of about $1^{\circ}$, the density of the ICM is $\sim 7.1 \times 10^{-4} \mathrm{~cm}^{-3}$, assuming a spherically symmetric and smooth distribution fitted with a double $\beta$-profile (Matsushita et al. 2002). The parameters used are given in Table 6. We get an upper limit on the local ram pressure of about $1820 \mathrm{~cm}^{-3}\left(\mathrm{~km} \mathrm{~s}^{-1}\right)^{2}=1.8 \times 10^{-11}$ dyne $\mathrm{cm}^{-2}$, assuming a deprojected orbital velocity of $1600 \mathrm{~km} \mathrm{~s}^{-1}$ for IC 3418 . The time evolution of the ram pressure exerted on the disk corresponds to a profile along an orbit of IC 3418 that is consistent with its observed plane of the sky position with respect to the Virgo cluster center and line-of-sight velocity. The projected tail direction was assumed to indicate the current direction of motion in the plane of the sky. We have studied orbits with perito apocenter ratios from about 1:5 to 1:20 that are characteristic for radial orbits in galaxy clusters (Ghigna et al. 1998; Boselli \& Gavazzi 2006; Vollmer 2009). As a fiducial orbit we have chosen an orbit that brings IC 3418 currently almost to pericenter, about $275 \mathrm{kpc}$ from the cluster center, with the plane of the sky velocity components $v_{x}=v_{y}=820 \mathrm{~km} \mathrm{~s}^{-1}$. The total 3D velocity is thus $\sim 1600 \mathrm{~km} \mathrm{~s}^{-1}$. The gravitational potential of the Virgo cluster was approximated by the spherically symmetric potential of the NFW dark matter halo (Navarro et al. 1996) with parameters specified in Table 6.

In Figs. 7 and 8, the vertical distance behind the disk to which parcels with different column densities occurring at different radii (up to $5 \mathrm{kpc}$; the $R_{25}$ major axis radius of IC 3418 is $\sim 3.6 \mathrm{kpc}$; see Table 1) can get in our model are shown for two time moments along the ram pressure event: 100 Myr before the peak and $100 \mathrm{Myr}$ after the peak. In the pre-peak situation, only parcels with $\Sigma_{\text {ISM }}<15 M_{\odot} \mathrm{pc}^{-2}$ can be shifted (not necessarily stripped) to at least $20 \mathrm{kpc}$, i.e., to distances comparable to the projected length of the tail of IC 3418 . From the nucleus of the galaxy, parcels with $\Sigma_{\text {ISM }} \approx 5-10 M_{\odot} \mathrm{pc}^{-2}$ can get to such distances. In the post-peak situation, more material is accelerated to larger distances - parcels from outer disk radii with $\Sigma_{\text {ISM }}$ up to about $35 M_{\odot} \mathrm{pc}^{-2}$, and from inner radii with $\Sigma_{\mathrm{ISM}}<15 M_{\odot} \mathrm{pc}^{-2}$, can get to $z \sim 20 \mathrm{kpc}$.

Figures 7 and 8 thus suggest that in our simulation lowdensity (H I-like) gas is stripped from the galaxy quite easily and can get to large distances; in the post-peak situation, all the gas with $\Sigma_{\text {ISM }} \lesssim 5 M_{\odot} \mathrm{pc}^{-2}$ gets to $z>100 \mathrm{kpc}$. Between the inner and outer radii, there is a large difference in the column density of parcels that can be moved to a certain distance. Denser gas parcels are thus difficult to strip substantially.

Figures 9 and 10 depict the vertical velocities relative to the galaxy to which ISM elements can be accelerated depending on their column density, for both the pre-peak and post-peak stages. Only low-density stripped parcels can reach high velocities in the pre-peak phase and the situation does not change much in the post-peak phase where elements with column densities $<15 M_{\odot} \mathrm{pc}^{-2}$ can occur at about $400 \mathrm{~km} \mathrm{~s}^{-1}$ relative to the galaxy. This excludes the possibility that some weak features noted in the spectra of the K4 and K6 tail positions in Sect. 3.2 may correspond to a real $\mathrm{CO}$ emission.

In Fig. 11 we show the time evolution of the stripping radii of ISM parcels with different column densities. The stripping radius of a disk gas component with some $\Sigma_{\text {ISM }}$ corresponds to a radius outside which it is released from the galaxy's potential $\left(E_{\mathrm{tot}}=E_{\mathrm{kin}}+E_{\mathrm{pot}}>0\right)$. Figure 11 provides a timescale for the stripping along our fiducial orbit: H I-like parcels (modeled with a Miyamoto-Nagai profile with $\Sigma_{0}=10 M_{\odot} \mathrm{pc}^{-2}$ that yields $\Sigma_{R=5 \mathrm{kpc}}=0.3 M_{\odot} \mathrm{pc}^{-2}$ ) start to be released from the outer disk by a weak ram pressure at cluster outskirts. It takes about $1 \mathrm{Gyr}$ before they are removed completely from $R=3 \mathrm{kpc}$ to the center of the galaxy. Denser gas parcels, with $\Sigma_{\text {ISM }}$ up to $\sim 20 \mathrm{M}_{\odot} \mathrm{pc}^{-2}$, can also be stripped throughout the disk. This happens on shorter timescales of 100-200 Myr from $R=3 \mathrm{kpc}$ to $0 \mathrm{kpc}$ as higher ram pressure arising only closer to the pericenter is required. For yet higher values of the column density the stripping radius starts 


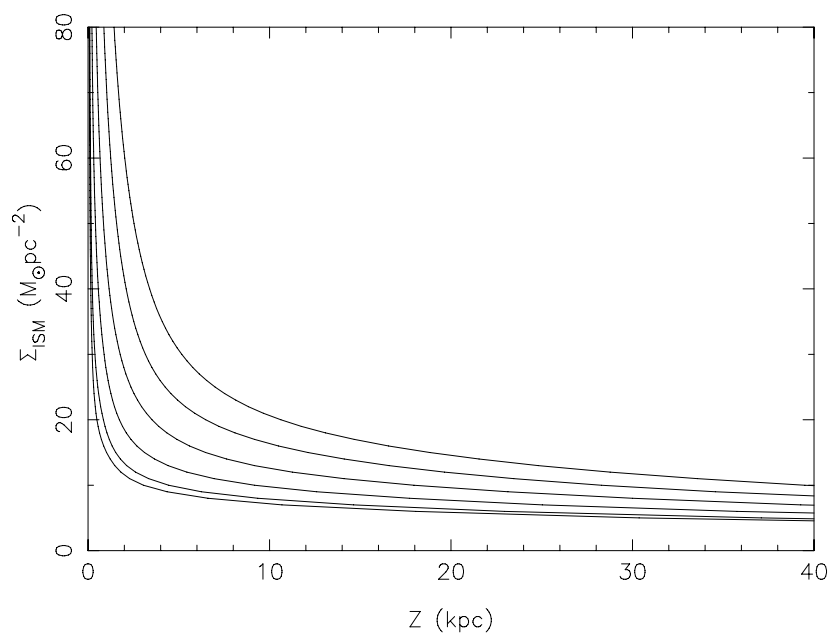

Fig. 7. Pre-peak stripping: vertical distances that ISM elements can reach with different column densities from radii 0-5 kpc (with $1 \mathrm{kpc}$ step; from left to right) in our model 100 Myr before the peak ram pressure.

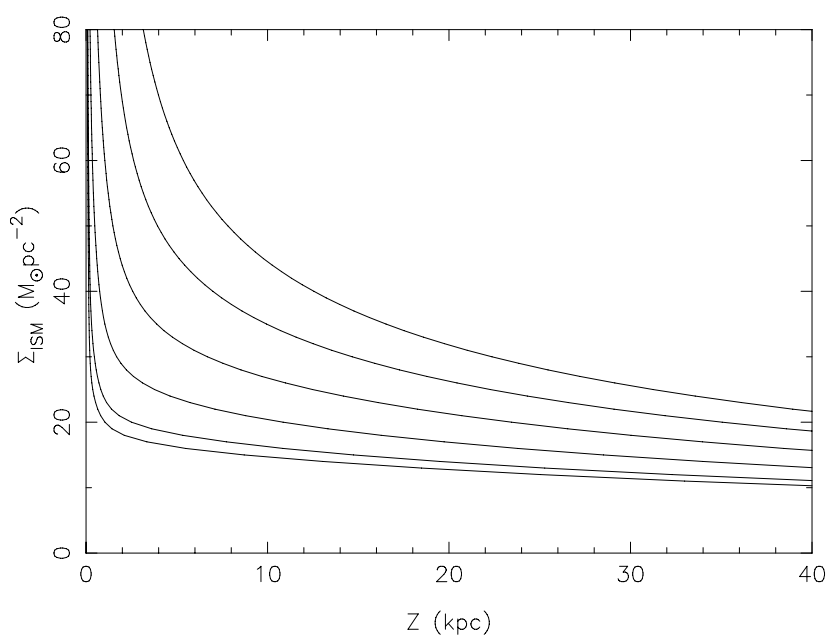

Fig. 8. Post-peak stripping. Same as in Fig. 7, but 100 Myr after the peak ram pressure.

to increase and subsequently approaches the outer disk radius. This happens at $\Sigma_{\text {ISM }} \sim 50 M_{\odot} \mathrm{pc}^{-2}$ which suggests that parcels with densities at this or a higher level can never be stripped from the galaxy by ram pressure alone.

Our simulation results are consistent with the results of spectral modeling of observations which suggested that star formation in the main body of IC 3418 stopped about 200-300 Myr ago, on a timescale of less than $\sim 70$ Myr from the outside in (Kenney et al., in prep.). Some of the denser gas in Fig. 11 is released only after maximum ram pressure, but this is part of material that had already been pushed out of the disk plane to the halo region, and will eventually fall back to the disk (see Jáchym et al. 2009, for details). Although our calculations depend on the adopted values of the free orbital parameters not constrained by observations and on the total mass of the galaxy, the results are consistent with what is expected from other simulations and existing observations of galaxies in clusters of the size of Virgo (Kenney \& Young 1989; Boselli et al. 2002; Fumagalli \& Gavazzi 2008): it is not possible to directly strip the dense (molecular) clouds, so they must have been removed from IC 3418 in some other way that we will discuss later.

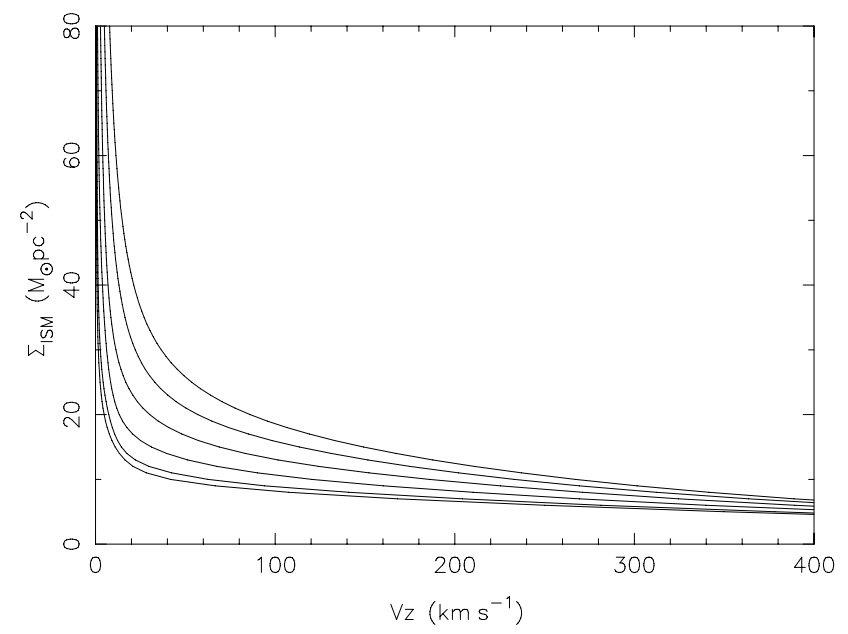

Fig. 9. Pre-peak stripping: vertical velocities to which ISM elements can be accelerated with different column densities from radii $0-5 \mathrm{kpc}$ (with $1 \mathrm{kpc}$ step; from left to right) in our model $100 \mathrm{Myr}$ before the peak ram pressure.

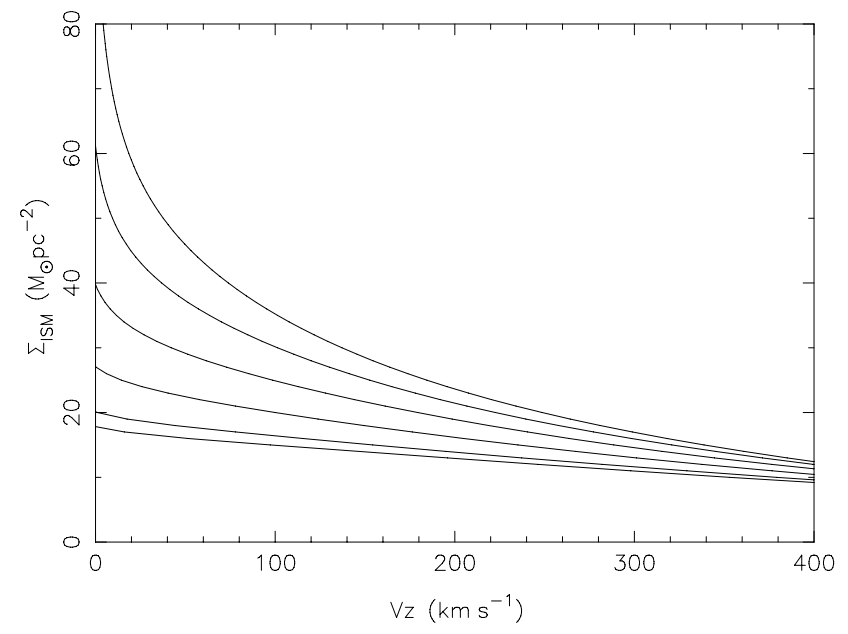

Fig. 10. Post-peak stripping. Same as in Fig. 9, but $100 \mathrm{Myr}$ after the peak ram pressure.

\section{Discussion}

\subsection{Central bulk of molecular gas}

If our marginal detection of $\mathrm{CO}$ emission in the $\mathrm{B} 1$ pointing is real, it corresponds to gas concentrated in the nuclear region. The possible survival of dense gas in the galaxy core (central few arcseconds $=$ few hundred pc) could either be because it is the center of the galaxy, the deepest part of the potential well from which it is most difficult to remove gas, or because the surface density of molecular gas is higher than the rest of the ISM, or both. While some molecular gas in the nucleus has apparently survived the stripping, there is no ongoing star formation in this gas. This might be due to a gas density that is below a threshold needed for star formation. The detected gas survived $\sim 200 \mathrm{Myr}$ after the cessation of star formation (Kenney et al., in prep.) that is presumably due to removal of most of the ISM.

The survival of molecular gas in the very central region of an otherwise gas-stripped dwarf irregular galaxy could be relevant for the formation of nucleated dwarf elliptical galaxies. Many cluster dEs have distinct nuclear star clusters, and it is not understood why some dEs have them and others do not. A surviving central gas reservoir may make it possible to form a nuclear 


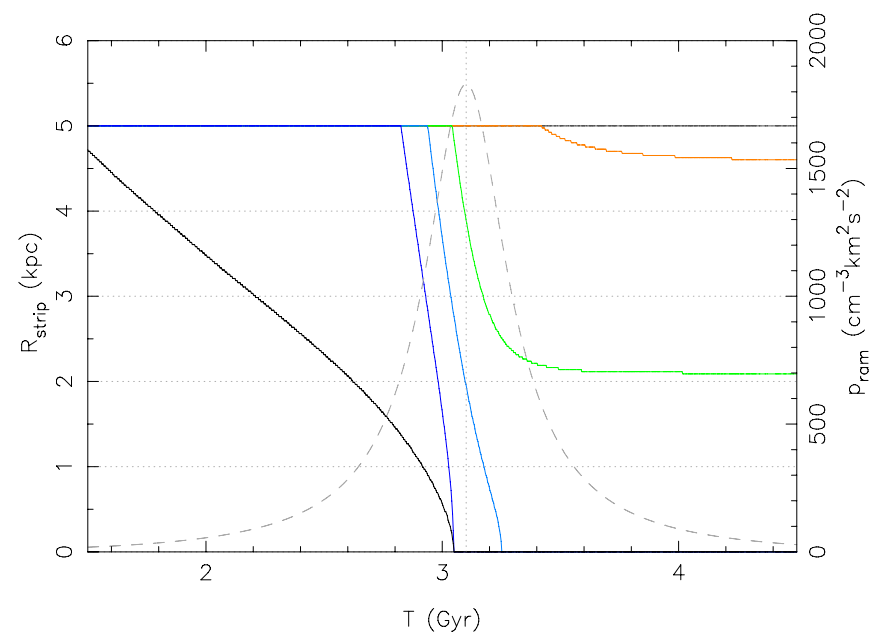

Fig. 11. Time evolution of stripping radii of ISM parcels with different column densities: H I-like (radius-dependent Miyamoto-Nagai profile with $\Sigma_{0}=10 M_{\odot} \mathrm{pc}^{-2}$ which yields $\Sigma_{R=5 \mathrm{kpc}}=0.3 M_{\odot} \mathrm{pc}^{-2}$ ), and constant $\Sigma_{\text {ISM }}=10,15,25$, and $50 M_{\odot} \mathrm{pc}^{-2}$ (from left to right) in our model. Ram pressure time profile along our model orbit is shown with the dashed line.

star cluster even after the galaxy is mostly gas-stripped. Also, as shown in numerical simulations, high-density clouds may lose angular momentum through interaction with the ICM and drift toward the center of the galaxy (Tonnesen \& Bryan 2009).

The heliocentric central velocity of the marginally detected central CO line is about $100 \mathrm{~km} \mathrm{~s}^{-1}$ (see Sect. 3.1). From Keck spectra, Kenney et al. (in prep.) measured a value of $170 \pm$ $10 \mathrm{~km} \mathrm{~s}^{-1}$ for the central velocity of the stars in the galaxy. The galaxy's rotation is presumably too small to account for such a velocity difference. Moreover, acceleration due to ram pressure would push the gas toward a higher radial velocity. On the other hand, if dense gas clouds can fall back into the galaxy after they condense in the tail and then become decoupled from ram pressure, they would have blue-shifted radial velocities relative to the central stellar disk. This behavior was noted in ram pressure stripping simulations of Kapferer et al. (2009) and could work in IC 3418. Future more sensitive $\mathrm{CO}$ observations could resolve the origin of the observed mismatch between the velocities of the stellar and molecular components.

\subsection{Molecular gas and star formation in the tail}

To assess the current molecular content of the tail of IC 3418, we inquire how much molecular gas is needed for the amount of star formation in the tail observed. Using the definition of GALEX $\mathrm{AB}$ magnitudes ${ }^{6}$, the total FUV luminosity of the tail is about $3.6 \times 10^{25} \mathrm{erg} \mathrm{s}^{-1} \mathrm{~Hz}^{-1}$ (Fumagalli et al. 2011). Making the assumption of a continuous star formation over the last $\sim 100 \mathrm{Myr}$, Eq. (2) predicts a SFR of $\sim 3.4 \times 10^{-3} M_{\odot} \mathrm{yr}^{-1}$. The total $\mathrm{H} \alpha$ luminosity of the knots in the outer half of the tail of about $2.4 \times 10^{38} \mathrm{erg} \mathrm{s}^{-1}$ (Fumagalli et al. 2011) corresponds to a SFR of $\sim 1.3 \times 10^{-3} M_{\odot} \mathrm{yr}^{-1}$ over the last $\sim 10 \mathrm{Myr}$ (Kennicutt \& Evans 2012) ${ }^{7}$. The difference in SFR estimates is not large and may be mainly because $\mathrm{H} \alpha$ emission is restricted to the outer part of the tail. Adopting a SFR in the tail of $\sim 3 \times 10^{-3} M_{\odot} \mathrm{yr}^{-1}$, and assuming a typical star formation timescale (or star formation efficiency $\operatorname{SFE}\left(\mathrm{H}_{2}\right)=\Sigma_{\mathrm{SFR}} / \Sigma_{\mathrm{H}_{2}}$ ) of $\sim 2$ Gyr (e.g., Bigiel et al. 2008; Leroy et al. 2013) we can deduce the mass of molecular

\footnotetext{
$m_{\mathrm{AB}}=-2.5 \log \left(f_{v}\left[\mathrm{erg} \mathrm{cm}^{-2} \mathrm{~s}^{-1} \mathrm{~Hz}^{-1}\right]\right)-48.6$.

$7 \log \operatorname{SFR}\left(M_{\odot} \mathrm{yr}^{-1}\right)=\log L_{\mathrm{H} \alpha}\left(\mathrm{erg} \mathrm{s}^{-1}\right)-41.27$.
}

gas expected to be occurring in the tail to be $6 \times 10^{6} M_{\odot}$. Our observed $(3 \sigma)$ upper limit on CO amount in the tail (see Table 4) is thus consistent with that predicted for a normal gas depletion timescale and normal X-factor. We would need more sensitive CO observations to show that either of these was non-standard.

Table 7 summarizes FUV and $\mathrm{H} \alpha$ luminosities for the main body and the three tail regions that we observed in $\mathrm{CO}$, and compares the corresponding SFRs. It shows that SFR estimates from the two tracers are in the knots similar, suggesting that indeed the difference in these quantities for the whole tail is because star formation stopped in parts of the tail. Applying our upper limit CO luminosities in the observed regions, we can estimate from the $\mathrm{H} \alpha$ SFRs lower limit values on the CO-to- $\mathrm{H}_{2}$ conversion factor $\alpha=\tau_{\text {dep }} \times \mathrm{SFR} / L_{\mathrm{CO}}$, assuming a constant depletion time $\tau_{\text {dep }}=2$ Gyr. The resulting limits are consistent with the Galactic value $\alpha_{\mathrm{CO}} \sim 4.4 M_{\odot} \mathrm{pc}^{-2}\left(\mathrm{~K} \mathrm{~km} \mathrm{~s}^{-1}\right)^{-1}$. Vollmer et al. (2012) and Boissier et al. (2012) determined that the star formation efficiency in the gas stripped tails of Virgo galaxies with respect to the available amount of atomic gas was at least 10 times lower than within the galaxies. However, Vollmer et al. (2012) found normal values of the SFE with respect to molecular content in the extraplanar regions of the galaxies.

\subsection{Where is the gas?}

Although dwarf galaxies are gas-rich systems, in IC 3418 practically no gas, neither atomic $\mathrm{H}$ I nor molecular $\mathrm{H}_{2}$, is observed. No H I is detected in the body and the tail, with fairly sensitive upper limits, $M_{\mathrm{H}}<8 \times 10^{6} M_{\odot}$ (Chung et al. 2009). This is $\sim 1 \%$ of the H I mass expected in a typical late type dwarf galaxy with the optical size or $H$-band luminosity of IC 3418 . We have possibly found only a small amount of molecular gas $M_{\mathrm{H}_{2}} \simeq 10^{6} M_{\odot}$ in the central $11^{\prime \prime}$ of the main body, and only upper limits $\left(M_{\mathrm{H}_{2}}<10^{7} M_{\odot}\right.$ assuming standard CO-to- $\mathrm{H}_{2}$ conversion) outside the central region and in the tail. While our upper limits are not sensitive enough to clearly demonstrate a lack of molecular gas in the tail (see Sect. 8.2), there is very likely a large amount of molecular gas missing in the main body. Although this is consistent with the observed absence of any ongoing star formation in the body of the galaxy, the question arises of what happened to the molecular gas that once occurred in IC 3418.

Calculations in the previous section have shown that lowdensity (atomic) gas can easily be completely stripped by ram pressure from a model of IC 3418, which is consistent with the sensitive non-detection of any $\mathrm{H}$ I emission. Higher-density gas with $\Sigma_{\text {ISM }}$ up to $\sim 50 M_{\odot} \mathrm{pc}^{-2}$ then can be accelerated from outer radii only, while the densest (molecular) gas stays bound to the galaxy. However, stripping by dynamical pressure of the ICM is probably enhanced by other processes such as ablation of dense clouds disrupted by hydrodynamic instabilities (e.g., Quilis et al. 2000; Kapferer et al. 2009; Tonnesen \& Bryan 2009), or shockheating and dissociation of molecular clouds due to supersonic motion of IC 3418 through the Virgo ICM (e.g., Guillard et al. 2012). Moreover, the structure of the ISM in dwarf irregulars can be significantly influenced by stellar feedback and often contains up to $\sim \mathrm{kpc}$-scale shells and holes seen in H I (see, e.g., Zhang et al. 2012, and references therein), which could facilitate stripping of the ISM as was shown in numerical simulations of Quilis et al. (2000) with holey disks. Thanks to a shallow potential well and low stellar surface densities, the ISM in dwarfs can also have lower average surface densities than in large spirals. Besides these indirect processes, a very important effect may also come 
Table 7. Star formation rates in the observed tail regions and corresponding lower limits on local $\mathrm{CO}-$ to- $\mathrm{H}_{2}$ conversion factor.

\begin{tabular}{lcccccc}
\hline \hline Source & $\begin{array}{c}L_{\mathrm{FUV}} \\
\left(10^{39} \mathrm{erg} \mathrm{s}^{-1}\right)\end{array}$ & $\begin{array}{c}L_{\mathrm{H} \alpha} \\
\left(10^{37} \mathrm{erg} \mathrm{s}^{-1}\right)\end{array}$ & $\begin{array}{c}S F R_{\mathrm{FUV}} \\
\left(10^{-4} M_{\odot} \mathrm{yr}^{-1}\right)\end{array}$ & $\begin{array}{c}S F R_{\mathrm{H} \alpha} \\
\left(10^{-4} M_{\odot} \mathrm{yr}^{-1}\right)\end{array}$ & $\begin{array}{c}L_{\mathrm{CO}} \\
\left(10^{5} \mathrm{~K} \mathrm{~km} \mathrm{~s}^{-1} \mathrm{pc}^{2}\right)\end{array}$ & $\begin{array}{c}\alpha_{\mathrm{CO}} \\
\left(M_{\odot} \mathrm{pc}^{-2}\left(\mathrm{~K} \mathrm{~km} \mathrm{~s}^{-1}\right)^{-1}\right)\end{array}$ \\
\hline Disk & 139.3 & - & 62.2 & - & $<11$ & - \\
K4 & 7.7 & 8.0 & 3.5 & 4.3 & $<2.6$ & $>3.3$ \\
K6 & 8.6 & 5.2 & 3.8 & 2.8 & $<2.3$ & $>2.4$ \\
K8 & 4.6 & 2.7 & 2.1 & 1.5 & $<2.1$ & $>1.4$ \\
\hline
\end{tabular}

Notes. FUV and $\mathrm{H} \alpha$ luminosities come from Fumagalli et al. (2011). FUV- and H $\alpha$-based SFRs were calculated using the formula of Kennicutt \& Evans (2012). For the $\mathrm{X}$-factor estimates, a $2 \mathrm{Gyr} \mathrm{H}_{2}$ depletion time was assumed.

Table 8. Upper limit $(3 \sigma)$ masses (in $M_{\odot}$ ) of gas components issued from different observations of the tail.

\begin{tabular}{ccccc}
\hline \hline & $\mathrm{H}_{2}$ & $\mathrm{H} \mathrm{I}$ & $\mathrm{H} \alpha$ & X-rays \\
\hline$M_{\text {tail }}$ & $<1 \times 10^{7}$ & $<4 \times 10^{8}$ & $<4 \times 10^{8} f_{\mathrm{H} \alpha}^{1 / 2}$ & $<4 \times 10^{7} f_{\mathrm{X}}^{1 / 2}$ \\
Ref. & this work & $\mathrm{Ch09}$ & this work $^{a}$ & this work \\
\hline
\end{tabular}

Notes. Ch09 = Chung et al. (2009); ${ }^{(a)}$ Based on $\mathrm{H} \alpha$ surface limit from Kenney et al. (in prep.).

from the rather limited lifetimes of dense clouds $(<100 \mathrm{Myr})$ that are comparable to the typical timescales of ram pressure stripping. The molecular gas reservoir could not then be replenished by further condensation and molecularization of H I since that was removed from the galaxy. All these processes, when combined together, may have resulted in effective stripping of molecular ISM in IC 3418. As a result, IC 3418 may currently be virtually deficient of almost all gas except possibly in its very center.

\subsection{Gas phases in the tail}

Table 8 summarizes the upper limits on the amount of different gas phases observed in the tail. Once stripped, the gas has probably been heated to temperatures between that of $\mathrm{HI}$ and the ICM, as cooler gas in the tail mixes with hot ICM gas, or more dense gas has been condensing into star-forming clouds. The absence of bright X-ray emission (see Sect. 6) is likely because of a low gas content in the tail associated with its advanced evolutionary state and/or because of rather low thermal pressure of the ICM at the position of IC 3418 in the Virgo cluster. Compression of stripped gas by the surrounding ICM determines the temperature and density distribution of gas in the tail. If present, the hot gas component in the tail is thus probably too diffuse to be observable in X-rays. The actual upper limit mass is close to $\sim 4 \times 10^{7} M_{\odot}$ since the filling factor of the X-ray emitting gas is not expected to be much smaller than 1. Associated with the HII regions, there is clearly ionized gas in the tail, but it is difficult to reliably estimate its mass without knowing the gas density; however the ionized gas mass in $\mathrm{H}$ II regions is generally small. While no diffuse $\mathrm{H} \alpha$ emission has been detected, there could be significant mass associated with undetected diffuse $10^{4} \mathrm{~K}$ gas. We derive the upper mass limit on $\mathrm{H} \alpha$ emitting diffuse gas from the $2 \sigma$ limit of the $\mathrm{H} \alpha$ surface brightness of $1.36 \times 10^{-17} \mathrm{erg} \mathrm{s}^{-1} \mathrm{~cm}^{-2} \operatorname{arcsec}^{-2} \mathrm{ob}-$ served with WIYN $3.5 \mathrm{~m}$ telescope (Kenney et al., in prep.). This is $\sim 2.3$ times more sensitive than the $\mathrm{H} \alpha$ image presented by Fumagalli et al. (2011). If the $\mathrm{H} \alpha$ emission is considered to originate from the same cylindrical volume of the X-ray gas, assuming an electron temperature of $10^{4} \mathrm{~K}$ and case $\mathrm{B}$ of nebular theory, the mass limit of the $\mathrm{H} \alpha$-emitting gas is $2.8 \times 10^{8} f_{\mathrm{H} \alpha}^{1 / 2} M_{\odot}$, where $\mathrm{f}_{\mathrm{H} \alpha}$ is the filling factor of the $\mathrm{H} \alpha$ gas. Numerical simulations of ram pressure stripping indicated that bright $\mathrm{H} \alpha$ emission is produced at the edges of dense neutral clouds (Tonnesen et al. 2011), so the volume filling factor should be low. From numerical simulations of Tonnesen et al. (2011) with the value of ICM thermal pressure consistent with that for ESO 137-001 (their Fig. 2, left panel), it follows that the typical local density of the $\mathrm{H} \alpha$ gas in the ESO 137-001 tail is $\sim 1 \mathrm{~cm}^{-3}$. Since the rms electron density observed in the tail of ESO 137-001 is $\sim 0.045 \mathrm{~cm}^{-3}$ (Sun et al. 2007), the filling factor, defined as the ratio of the average density and local density, is estimated to be about $5 \times 10^{-2}$. Thus, a reasonable upper limit on the amount of $\mathrm{H} \alpha$ emitting gas in the tail of IC 3418 would be about $6 \times 10^{7} M_{\odot}$. Considering the atomic component of the gas in the tail, the mass upper limit is $\sim 4 \times 10^{8} M_{\odot}$ following from the upper limit of $8 \times 10^{6} M_{\odot}$ per beam $\left(F W H M \sim 16^{\prime \prime}\right.$, Chung et al. 2009), and assuming the same tail dimensions used above for the X-ray mass estimate (see Sect. 6). The actual amount of the $\mathrm{HI}$ in the tail may be much lower than the limit since denser clouds have probably condensed into stars while the diffuse gas had been stripped to large distances (very likely exceeding the observed length of the tail), as follows from the calculations in Sect. 7. Numerical simulations of Tonnesen et al. (2011) suggest that in the rather low thermal pressure environment surrounding IC 3418, the mass fractions of cool $\left(300 \mathrm{~K}<T<10^{4} \mathrm{~K}\right)$ and hot $\left(7 \times 10^{5} \mathrm{~K}<T<4 \times 10^{7} \mathrm{~K}\right)$ components in the stripped gas might be both of about $30 \%$ of the total stripped mass without much evolution as the tail ages from $100 \mathrm{Myr}$ to $250 \mathrm{Myr}$.

\subsection{Origin of star formation in the tail}

Galaxies with star forming tails are rare in the Virgo cluster. Extraplanar $\mathrm{H} \alpha$ emission was found in only a few ram pressure stripped galaxies (NGC 4522, NGC 4402, NGC 4330, NGC 4438, Kenney \& Koopmann 1999; Cortese et al. 2003; Abramson et al. 2011; Boselli et al. 2005, respectively) where it typically occurs only fairly close to the disk. Those are galaxies with typical (stellar) mass at least a factor of 10 higher than that of IC 3418. In IC 3418 the situation is different - a large number of young stars occur at quite large distances (up to $17 \mathrm{kpc}$ in projection) from the disk distributed in a straight, narrow tail. Chung et al. (2009) suggested that recent star formation in the tail occurred from compression of the stripped H I gas. Similarly, Hester et al. (2010) speculated that star formation in the wake was driven by turbulence that enhances density contrasts and thus aids gas cooling and condensation. A similar example of star formation in situ in the stripped gas is known in the Virgo dwarf galaxy VCC 1249 (Arrigoni Battaia et al. 2012) which is, however, stripped by the ISM of an elliptical galaxy (M49), whereas IC 3418 is stripped by the ICM in the Virgo cluster. 
Hydrodynamic AMR simulations of Tonnesen \& Bryan (2012) showed that star formation may occur in the ram pressure stripped tails when radiative cooling can overcome the heating from the surrounding ICM and thus is able to cool down the stripped ISM. Our simplified calculations in Sect. 7 have suggested that although molecular gas cannot be directly stripped by ram pressure from IC 3418, some higher density $\mathrm{H}$ I than is usual in larger spirals in Virgo $\left(\Sigma_{\text {ISM }}<25 M_{\odot} \mathrm{pc}^{-2}\right.$ within the inner $2 \mathrm{kpc}$ disk radius, and $<50 M_{\odot} \mathrm{pc}^{-2}$ in the outer disk radii) can be stripped thanks to a shallow potential of the galaxy and its proximity to the cluster center. It is then possible that these higherdensity clumps were easier to cool and compress by ram pressure together with the thermal pressure of the ICM, supporting their condensation. Moreover, because of the supersonic motion of IC 3418 through the ICM, the thermal pressure of the post-shock environment surrounding the stripped ISM within its Mach cone is probably higher than the pre-shock thermal pressure, which could also enhance condensation of the stripped material. In simulations, the stripped ISM fragments strongly behind the bow shock as a result of turbulence and Rayleigh-Taylor instability (e.g., Roediger et al. 2006). As shown, e.g., by Zhang et al. (2012), the inner disks in nearby dwarf irregulars have proportionally more cool gas than the outer disks. Leroy et al. (2008) indeed suggested that $\mathrm{H}_{2}$ dominates ISM of nearby dwarfs in the inner parts of their disks $\left(<0.25 r_{25}\right)$ and not at the outer radii. Then ram pressure stripped cool gas and denser clumps originate preferentially from the inner disk regions. The global head-tail morphology of IC 3418 with a narrow (and linear) tail extending behind a larger main body of the galaxy, would then be consistent with the proposed scenario.

\section{Conclusion}

We searched for cold molecular gas, as well as hot X-ray emitting gas, in IC 3418, a dwarf galaxy that is currently being transformed by ram pressure stripping from an irregular to an earlytype galaxy. It contains a prominent tail of young stars, a feature that is rare among other ram pressure stripped Virgo galaxies. Using the IRAM $30 \mathrm{~m}$ antenna, we searched for CO emission in five deep integrations in the main body of the galaxy and in its gas-stripped tail. New deep Chandra observations covered the whole system. The main results are:

1. A possible ${ }^{12} \mathrm{CO}(2-1) 5 \sigma$ marginal detection suggested that about $1.2 \times 10^{6} M_{\odot}$ of molecular gas (assuming a standard Galactic CO-to- $\mathrm{H}_{2}$ conversion factor) is present in the central few arcseconds of IC 3418. Although CO(1-0) emission was not formally detected in the center of the galaxy, there is a $2 \sigma$ feature with the same velocity range as the $\mathrm{CO}(2-1)$ line, which is consistent with a weak emission diluted by the $\mathrm{CO}(1-0)$ large beam. An upper limit on molecular gas content in the whole main body of the galaxy is estimated to $<5 \times 10^{6} M_{\odot}$, assuming the standard Galactic CO-to- $\mathrm{H}_{2}$ conversion factor. A similar limit is obtained from the existing upper limits on FIR emission from dust. This is a factor of $\sim 20$ less than the original molecular gas content of the galaxy, as estimated from the UV-based SFR before quenching, and the typical gas depletion timescale in spirals of 2 Gyr. While some of this difference may arise from a nonstandard $\mathrm{CO}$-to- $\mathrm{H}_{2}$ conversion that could be $\sim 5$ times larger than Galactic, we think much of it is due to a true deficiency of molecular gas, given the lack of star formation in the main body.

2. Although the presence of H II regions in the tail suggests that there must be some dense (presumably molecular) gas in the tail, we have found only $(3 \sigma)$ upper limits of $<10^{6} M_{\odot}$ in the three observed points covering the outer tail regions. The estimated upper limit on the molecular gas content of the whole tail is $\sim 1 \times 10^{7} M_{\odot}$. This is an amount that is similar to the estimates based on the observed SFR averaged over the tail, assuming a normal gas depletion timescale of 2 Gyr. Thus with our current sensitivity we cannot claim that the relationship between the dense gas content and the SFR in the tail is unusual.

3. Very sensitive upper limits on the $0.2-5 \mathrm{keV}$ luminosity of the gas-stripped tail has been obtained with Chandra. The corresponding X-ray mass limit of the tail is $<3.7 \times$ $10^{7} f_{\mathrm{X}^{1 / 2}} M_{\odot}$ (where $f_{\mathrm{X}}$ is the filling factor of the X-ray emitting gas) which is a factor of $\sim 280$ less than in the tail of the Norma cluster ram pressure stripped galaxy ESO 137-001. The absence of a bright X-ray tail may be mainly due to a low gas content in the tail, associated with its advanced evolutionary state in which gas from the main body of the galaxy is no longer supplied to the tail. A rather low thermal pressure of the surrounding ICM in the Virgo cluster could also account for our non-detection. Existing AMR hydrodynamic numerical simulations would suggest that at least some soft X-ray emission should be observable in the tail of IC 3418; however, these models do not study the evolutionary state after the galaxy is fully stripped.

4. Our semi-analytic calculations have indicated that ISM parcels with rather high column densities up to $\sim 25-50 M_{\odot} \mathrm{pc}^{-2}$ (depending on the galactic radius) can be accelerated from the galaxy. However, molecular clouds with densities $>100 M_{\odot} \mathrm{pc}^{-2}$ cannot be directly stripped from the inner part of IC 3418 by the ram pressure in Virgo, even though this dwarf galaxy has a shallow galactic potential, and it may get close to the cluster center. This explains why some molecular gas could survive in the galaxy center. Moreover, the apparent loss of most of the molecular gas in the galaxy must be due to rather short lifetimes of dense clouds that are similar to stripping timescales, and also due to processes other than direct stripping, meaning some form of cloud destruction after the surrounding low density gas is stripped. Such processes may contain ablation, which includes Kelvin-Helmholtz instabilities, and self-destruction of the clouds by supersonic motion of the galaxy through the Virgo ICM.

5. The surviving central molecular gas reservoir makes it possible for a nuclear star cluster to eventually form, even after most of the galaxy's gas has been stripped.

6. We have suggested that ram pressure may act as an external agent that, together with the thermal pressure of the ICM and assisted by radiative cooling, compresses the stripped dense clumps and thus may trigger star formation in the tail of the galaxy.

Acknowledgements. We acknowledge support by the projects RVO:67985815, M100031203 of the Academy of Sciences of the Czech Republic, GA ČR P209/11/P699, and GA ČR P209/12/1795. Pavel Jáchym was a Fulbright fellow at Yale University in 2009-2010 when this project was prepared. Ming Sun is supported by NASA grants GO2-13102X, GO0-11145C, and GO1-12103A. We thank H. Crowl and S. Tonnesen for useful comments. The scientific results reported in this article are based in part on observations made by the Chandra X-ray Observatory. This research has made use of the NASA/IPAC Extragalactic Database (NED) which is operated by the Jet Propulsion Laboratory, California Institute of Technology, under contract with the National Aeronautics and Space Administration, and of the HyperLeda database (http://leda.univ-lyon 1. fr). 


\section{References}

Abramson, A., Kenney, J. D. P., Crowl, H. H., et al. 2011, AJ, 141, 164 Anders, E., \& Grevesse, N. 1989, Geochim. Cosmochim. Acta, 53, 197 Arrigoni Battaia, F., Gavazzi, G., Fumagalli, M., et al. 2012, A\&A, 543, A112 Asplund, M., Grevesse, N., Sauval, A. J., \& Scott, P. 2009, ARA\&A, 47, 481 Bell, E. F., \& de Jong, R. S. 2000, MNRAS, 312, 497

Bell, E. F., \& de Jong, R. S. 2001, ApJ, 550, 212

Bigiel, F., Leroy, A., Walter, F., et al. 2008, AJ, 136, 2846

Binggeli, B., Tammann, G. A., \& Sandage, A. 1987, AJ, 94, 251

Binney, J., \& Tremaine, S. 2008, Galactic Dynamics, 2nd edn. (Princeton University Press)

Boissier, S., Boselli, A., Duc, P.-A., et al. 2012, A\&A, 545, A142

Bolatto, A. D., Leroy, A. K., Rosolowsky, E., Walter, F., \& Blitz, L. 2008, ApJ, 686, 948

Boselli, A., \& Gavazzi, G. 2006, PASP, 118, 517

Boselli, A., Lequeux, J., \& Gavazzi, G. 2002, A\&A, 384, 33

Boselli, A., Boissier, S., Cortese, L., et al. 2005, ApJ, 623, L13

Boselli, A., Boissier, S., Cortese, L., \& Gavazzi, G. 2008, ApJ, 674, 742

Bothwell, M. S., Kennicutt, R. C., \& Lee, J. C. 2009, MNRAS, 400, 154

Chung, A., van Gorkom, J. H., Kenney, J. D. P., \& Vollmer, B. 2007, ApJ, 659, L115

Chung, A., van Gorkom, J. H., Kenney, J. D. P., Crowl, H., \& Vollmer, B. 2009, AJ, 138, 1741

Cortese, L., Gavazzi, G., Iglesias-Paramo, J., Boselli, A., \& Carrasco, L. 2003, A\&A, 401, 471

Cortese, L., Marcillac, D., Richard, J., et al. 2007, MNRAS, 376, 157

Cortese, L., Bendo, G. J., Boselli, A., et al. 2010, A\&A, 518, L63

Crowl, H. H., \& Kenney, J. D. P. 2006, ApJ, 649, L75

Crowl, H. H., Kenney, J. D. P., van Gorkom, J. H., \& Vollmer, B. 2005, AJ, 130, 65

Evans, A. S., Mazzarella, J. M., Surace, J. A., et al. 2005, ApJS, 159, 197

Feldmann, R., Gnedin, N. Y., \& Kravtsov, A. V. 2012, ApJ, 758, 127

Fumagalli, M., \& Gavazzi, G. 2008, A\&A, 490, 571

Fumagalli, M., Krumholz, M. R., Prochaska, J. X., Gavazzi, G., \& Boselli, A. 2009, ApJ, 697, 1811

Fumagalli, M., Gavazzi, G., Scaramella, R., \& Franzetti, P. 2011, A\&A, 528, A46

Gavazzi, G., Boselli, A., Donati, A., Franzetti, P., \& Scodeggio, M. 2003, A\&A, 400,451

Gavazzi, G., Boselli, A., van Driel, W., \& O’Neil, K. 2005, A\&A, 429, 439

Gavazzi, G., Boselli, A., Cortese, L., et al. 2006, A\&A, 446, 839

Ghigna, S., Moore, B., Governato, F., et al. 1998, MNRAS, 300, 146

Gil de Paz, A., Boissier, S., Madore, B. F., et al. 2007, ApJS, 173, 185

Guillard, P., Boulanger, F., Pineau des Forêts, G., et al. 2012, ApJ, 749, 158

Gunn, J. E., \& Gott, III, J. R. 1972, ApJ, 176, 1

Hester, J. A., Seibert, M., Neill, J. D., et al. 2010, ApJ, 716, L14

Hunter, D. A., Elmegreen, B. G., \& Ludka, B. C. 2010, AJ, 139, 447

Israel, F. P. 1997, A\&A, 328, 471

Jáchym, P., Palouš, J., Köppen, J., \& Combes, F. 2007, A\&A, 472, 5

Jáchym, P., Köppen, J., Palouš, J., \& Combes, F. 2009, A\&A, 500, 693

Kalberla, P. M. W., Burton, W. B., Hartmann, D., et al. 2005, A\&A, 440, 775

Kapferer, W., Sluka, C., Schindler, S., Ferrari, C., \& Ziegler, B. 2009, A\&A, 499, 87

Kenney, J. D. P., \& Koopmann, R. A. 1999, AJ, 117, 181

Kenney, J. D., \& Young, J. S. 1986, ApJ, 301, L13

Kenney, J. D. P., \& Young, J. S. 1989, ApJ, 344, 17

Kenney, J. D. P., van Gorkom, J. H., \& Vollmer, B. 2004, AJ, 127, 3361
Kennicutt, R. C., \& Evans, N. J. 2012, ARA\&A, 50, 531

Kewley, L. J., \& Ellison, S. L. 2008, ApJ, 681, 1183

Kirby, E. M., Koribalski, B., Jerjen, H., \& López-Sánchez, Á. 2012, MNRAS, 420, 2924

Komugi, S., Yasui, C., Kobayashi, N., et al. 2011, PASJ, 63, L1

Koopmann, R. A., \& Kenney, J. D. P. 2004, ApJ, 613, 851

Kormendy, J., \& Bender, R. 2012, ApJS, 198, 2

Leitherer, C., Schaerer, D., Goldader, J. D., et al. 1999, ApJS, 123, 3

Leroy, A., Bolatto, A. D., Simon, J. D., \& Blitz, L. 2005, ApJ, 625, 763

Leroy, A., Bolatto, A., Stanimirovic, S., et al. 2007a, ApJ, 658, 1027

Leroy, A., Cannon, J., Walter, F., Bolatto, A., \& Weiss, A. 2007b, ApJ, 663, 990

Leroy, A. K., Walter, F., Brinks, E., et al. 2008, AJ, 136, 2782

Leroy, A. K., Walter, F., Sandstrom, K., et al. 2013, AJ, 146, 19

Lisenfeld, U., \& Ferrara, A. 1998, ApJ, 496, 145

Maloney, P., \& Black, J. H. 1988, ApJ, 325, 389

Matsushita, K., Belsole, E., Finoguenov, A., \& Böhringer, H. 2002, A\&A, 386, 77

Mei, S., Blakeslee, J. P., Côté, P., et al. 2007, ApJ, 655, 144

Mizuno, N., Rubio, M., Mizuno, A., et al. 2001, PASJ, 53, L45

Mo, H., van den Bosch, F. C., \& White, S. 2010, Galaxy Formation and Evolution (Cambridge University Press)

Navarro, J. F., Frenk, C. S., \& White, S. D. M. 1996, ApJ, 462, 563

Obreschkow, D., \& Rawlings, S. 2009, MNRAS, 394, 1857

Paturel, G., Petit, C., Prugniel, P., et al. 2003, A\&A, 412, 45

Pettini, M., \& Pagel, B. E. J. 2004, MNRAS, 348, L59

Pineda, J. L., Goldsmith, P. F., Chapman, N., et al. 2010, ApJ, 721, 686

Poggianti, B. M., Smail, I., Dressler, A., et al. 1999, ApJ, 518, 576

Quilis, V., Moore, B., \& Bower, R. 2000, Science, 288, 1617

Randall, S., Nulsen, P., Forman, W. R., et al. 2008, ApJ, 688, 208

Roediger, E., \& Hensler, G. 2005, A\&A, 433, 875

Roediger, E., Brüggen, M., \& Hoeft, M. 2006, MNRAS, 371, 609

Schindler, S. 1999, A\&A, 349, 435

Schruba, A., Leroy, A. K., Walter, F., et al. 2012, AJ, 143, 138

Shibata, R., Matsushita, K., Yamasaki, N. Y., et al. 2001, ApJ, 549, 228

Smith, R. J., Lucey, J. R., Hammer, D., et al. 2010, MNRAS, 408, 1417

Sun, M., \& Vikhlinin, A. 2005, ApJ, 621, 718

Sun, M., Donahue, M., \& Voit, G. M. 2007, ApJ, 671, 190

Sun, M., Donahue, M., Roediger, E., et al. 2010, ApJ, 708, 946

Taylor, C. L., Kobulnicky, H. A., \& Skillman, E. D. 1998, AJ, 116, 2746

Tonnesen, S., \& Bryan, G. L. 2009, ApJ, 694, 789

Tonnesen, S., \& Bryan, G. L. 2010, ApJ, 709, 1203

Tonnesen, S., \& Bryan, G. L. 2012, MNRAS, 2609

Tonnesen, S., Bryan, G. L., \& Chen, R. 2011, ApJ, 731, 98

Tremonti, C. A., Heckman, T. M., Kauffmann, G., et al. 2004, ApJ, 613, 898

Tuffs, R. J., Popescu, C. C., Pierini, D., et al. 2002, ApJS, 139, 37

van Gorkom, J. H. 2004, Clusters of Galaxies: Probes of Cosmological Structure and Galaxy Evolution, 305

Vollmer, B. 2009, A\&A, 502, 427

Vollmer, B., Cayatte, V., Balkowski, C., \& Duschl, W. J. 2001, ApJ, 561, 708

Vollmer, B., Braine, J., Pappalardo, C., \& Hily-Blant, P. 2008, A\&A, 491, 455

Vollmer, B., Wong, O. I., Braine, J., Chung, A., \& Kenney, J. D. P. 2012, A\&A, 543, A33

Weżgowiec, M., Vollmer, B., Ehle, M., et al. 2011, A\&A, 531, A44

Woudt, P. A., Kraan-Korteweg, R. C., Lucey, J., Fairall, A. P., \& Moore, S. A. W. 2008, MNRAS, 383, 445

Yoshida, M., Yagi, M., Komiyama, Y., et al. 2008, ApJ, 688, 918

Young, J. S., Xie, S., Tacconi, L., et al. 1995, ApJS, 98, 219

Zhang, H.-X., Hunter, D. A., \& Elmegreen, B. G. 2012, ApJ, 754, 29 